\title{
Preliminary Design Study for an Eddy Current Flow Meter for the Versatile Test Reactor
}

Approved for public release. Distribution is unlimited.

\section{OAK RIDGE NATIONAL LABORATORI} MANAGED BY UT BATTELLE FOR US. DEI

Sacit M. Cetiner Kellen M. Oleksak R. J. Bruce Warmack Michael J. Roberts Milton Nance Ericson

Aly Fathy (University of Tennessee)

June 2019 


\section{DOCUMENT AVAILABILITY}

Reports produced after January 1, 1996, are generally available free via US Department of Energy (DOE) SciTech Connect.

Website: http://www.osti.gov

Reports produced before January 1, 1996, may be purchased by members of the public from the following source:

National Technical Information Service

5285 Port Royal Road

Springfield, VA 22161

Telephone: 703-605-6000 (1-800-553-6847)

TDD: $703-487-4639$

Fax: 703-605-6900

E-mail: info@ntis.gov

Website: http://classic.ntis.gov/

Reports are available to DOE employees, DOE contractors, Energy Technology Data Exchange representatives, and International Nuclear Information System representatives from the following source:

Office of Scientific and Technical Information

PO Box 62

Oak Ridge, TN 37831

Telephone: 865-576-8401

Fax: 865-576-5728

E-mail: report@osti.gov

Website: http://www.osti.gov/contact.html

This report was prepared as an account of work sponsored by an agency of the United States Government. Neither the United States Government nor any agency thereof, nor any of their employees, makes any warranty, express or implied, or assumes any legal liability or responsibility for the accuracy, completeness, or usefulness of any information, apparatus, product, or process disclosed, or represents that its use would not infringe privately owned rights. Reference herein to any specific commercial product, process, or service by trade name, trademark, manufacturer, or otherwise, does not necessarily constitute or imply its endorsement, recommendation, or favoring by the United States Government or any agency thereof. The views and opinions of authors expressed herein do not necessarily state or reflect those of the United States Government or any agency thereof. 
Reactor and Nuclear Systems Division

\title{
PRELIMINARY DESIGN STUDY FOR AN EDDY CURRENT FLOW METER FOR THE VERSATILE TEST REACTOR
}

\author{
Sacit M. Cetiner \\ Kellen M. Oleksak \\ R. J. Bruce Warmack \\ Michael J. Roberts \\ Milton Nance Ericson \\ Aly Fathy (University of Tennessee,Knoxville)
}

Date Published: June 28, 2019

Prepared by

OAK RIDGE NATIONAL LABORATORY

Oak Ridge, TN 37831-6283

managed by

UT-Battelle, LLC

for the

US DEPARTMENT OF ENERGY

under contract DE-AC05-00OR22725 



\section{CONTENTS}

LIST OF FIGURES $\ldots \ldots \ldots \ldots \ldots \ldots \ldots \ldots \ldots$

LIST OF TABLES $\ldots \ldots \ldots \ldots \ldots \ldots \ldots \ldots \ldots$ vii

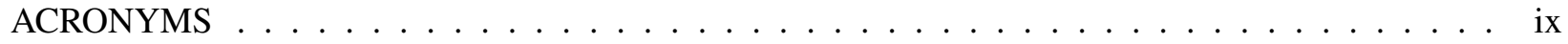

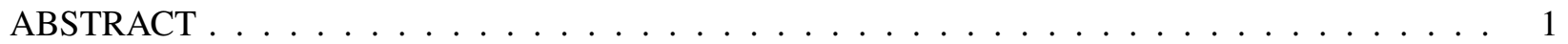

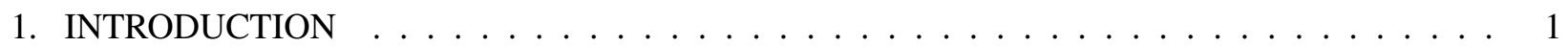

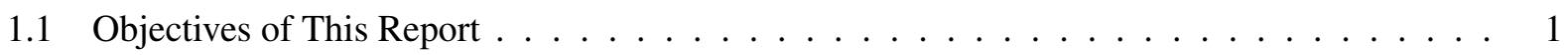

1.2 Organization of This Report $\ldots \ldots \ldots \ldots \ldots \ldots \ldots$

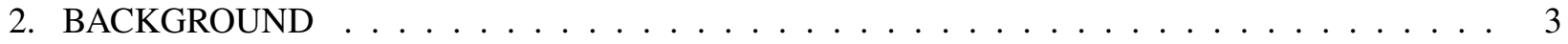

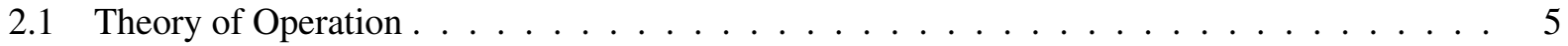

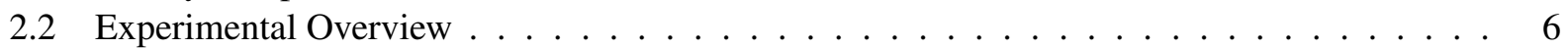

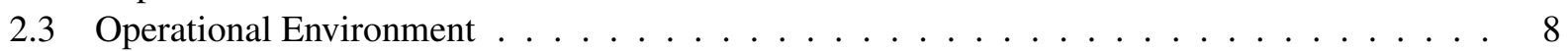

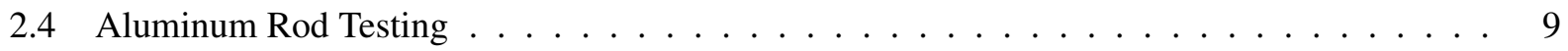

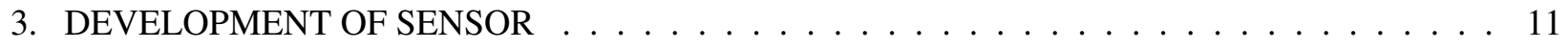

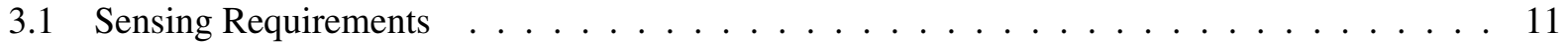

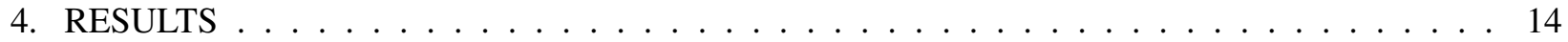

$4.1 \quad$ Numerical Simulations: Aluminum $\operatorname{Rod} \ldots \ldots \ldots$. . . . . . . . . . . . . . . 14

4.2 Numerical Simulations: Mercury and Galinstan . . . . . . . . . . . . . . . . . 20

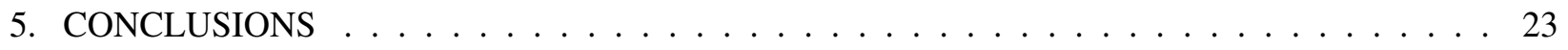

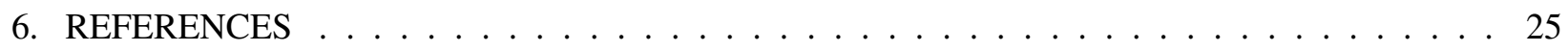

APPENDIX A. MAXWELL'S EQUATIONS FOR MHD . . . . . . . . . . . . . . . . . . A-1 



\section{LIST OF FIGURES}

$1 \quad$ ECFM development process. . . . . . . . . . . . . . . . . . . . 2

2 RDT specification ECFM probe, RDT C 4-7T [June 1973] . . . . . . . . . . . . . . . . 3

3 ECFM operation and wiring, RDT C 4-7T [June 1973] . . . . . . . . . . . . . . . . 4

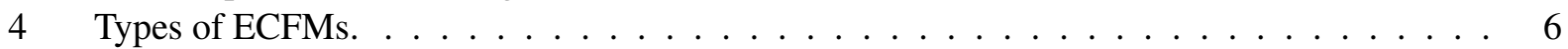

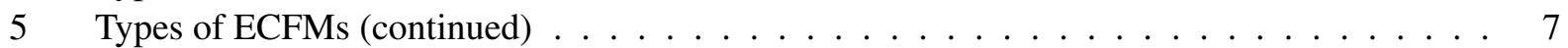

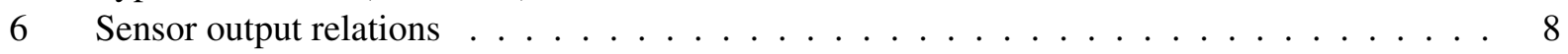

$7 \quad$ ECFM core placement, Nutt and Stringer [March 1973]. . . . . . . . . . . . . . . . 8

8 FOTA and ECFM placement, Nutt and Stringer [March 1973] . . . . . . . . . . . . . . 9

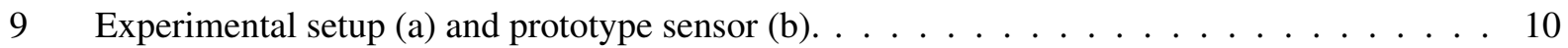

10 RDT specification ECFM probe, RDT C 4-7T [June 1973] . . . . . . . . . . . . . . . . 12

11 Probe example with fill gas, RDT C 4-7T [June 1973]. . . . . . . . . . . . . . . . . . . . 13

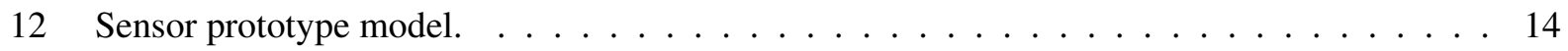

13 Bobbin design. . . . . . . . . . . . . . . . . . . . . . . . . . . 14

14 Sensitivity curves for $6061 \mathrm{~T}$ aluminum rod $\ldots \ldots \ldots \ldots \ldots$

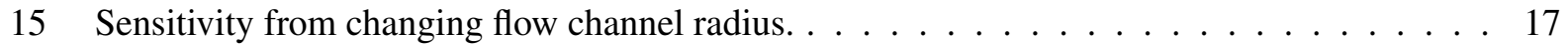

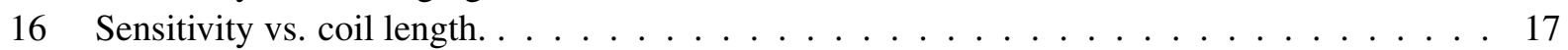

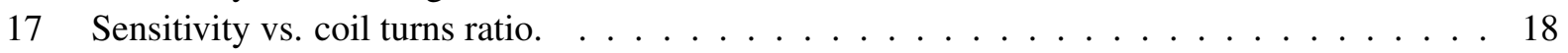

18 Magnetic flux density for $B_{z}(r, z)$ and $B_{r}(r, z) \ldots \ldots \ldots \ldots \ldots \ldots$

19 Comparison of galinstan and mercury velocity profiles and magnetic flux density $B_{r}(r, z)$. . 21

20 Comparison of frequency response for Mercury and Galinstan. . . . . . . . . . . . . . 22

21 Comparison of Gglinstan and Mercury sensor output voltage as a function of velocity. . . . . 22 



\section{LIST OF TABLES}

$1 \quad$ RDT material specifications . . . . . . . . . . . . . . . . . . . . . 12

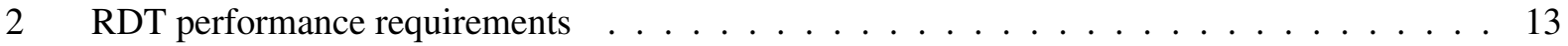

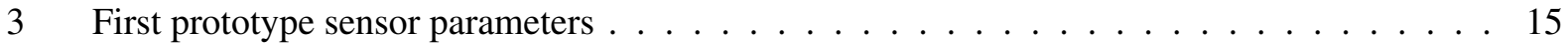

4 Sensor material properties $(@ 293 \mathrm{~K}) \ldots \ldots \ldots \ldots \ldots \ldots$

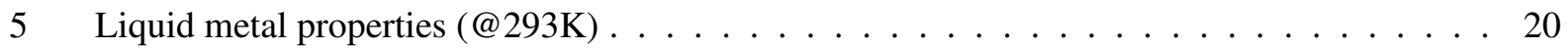



ACRONYMS

BOP Bipolar Operational Amplifier

ECFM Eddy Current Flow Meter

emf electromotive force

EM Electromagnetic

FFTF Fast Flux Test Facility

FOTA Fuels Open Test Assembly

HEDL Hanford Engineering and Development Laboratory

LVDT Linear Variable Differential Transformer

MHD Magnetohydrodynamics

ORNL Oak Ridge National Laboratory

RDT Research and Development Transmittal

SNR Signal-to-Noise Ratio

SS Stainless Steel 



\begin{abstract}
The eddy current flow meter (ECFM) serves the purpose of monitoring velocities and temperatures of conductive liquid coolant surrounding nuclear reactor cells. The ECFM can also detect voids in two-phase liquid metal flows. Measurement of this flow in reactor cells can greatly improve the accuracy of the empirical formulas used for fluid dynamics near the reactor core, as well as, aid in mapping any non-uniformities in fluid flow, its temperature estimation and phase. With modeling and experimental verification, an extensible ECFM for multiple facilities and environments can lead to an understanding of the liquid coolant flow; while saving cost of calibration and development. This can be done by characterizing the ECFM performance based on its parameters and then optimizing a sensor for a given use case. Simulation results using an ECFM and an aluminum rod medium are presented and discussed herein. Additionally, liquid metal simulations for Mercury and Galinstan will compare the ECFM behavior for different liquid velocities and excitation frequencies. After sensor calibration curves are generated and experimentally verified, ECFMs can be developed and deployed using the model introduced.
\end{abstract}

\title{
1. INTRODUCTION
}

\subsection{Objectives of This Report}

Development and testing of the ECFM aims to characterize optimal sensor parameters and performance for a given environment. This includes but is not limited to, sensor size, sensitivity, maintainability, reliability and duration of use. Overall objectives of this work are as follows:

- Produce a model which accurately describes a sensor's performance in a proposed environment.

- Appropriately optimize ECFM sensors based on simulation and experimental results.

- Generate calibration curves which yield the velocity of the liquid coolant being monitored by the sensor.

The stages of the project that guide the above objectives are shown in Figure 1.

Development of the ECFM is guided by the need for simulation, experimental testing, verification, and model updating. Once the model accurately represents the experimental behavior, calibration curves related to the sensor's output can be used to determine velocity of the liquid flow and temperature. Temperature sensing of the ECFM is not a part of this report and will take place when environmental conditions are more accurately modeled and reproduced in the lab. It is imperative that the physical model guides the enhancement of the modeled ECFM to make it an accurate system component in reactor simulations.

This report characterizes the sensor in relation to the dry testing stage, and it also reports experimental results from these tests. Simulation results for liquid testing are presented in a general manner, as the experimental setup is yet to be determined. The dry testing stage is useful for confirming equipment operation, data processing, and overall lab setup. Starting with a low-temperature, non-toxic solid rod avoids the safety-issues associated with high-temperatures and toxic liquid metals. Additionally, the solid rod is more accessible and allows quicker modifications to the experiment to hone in on sensor qualities before moving to a liquid loop stage which is logistically more complex and more expensive. Once 


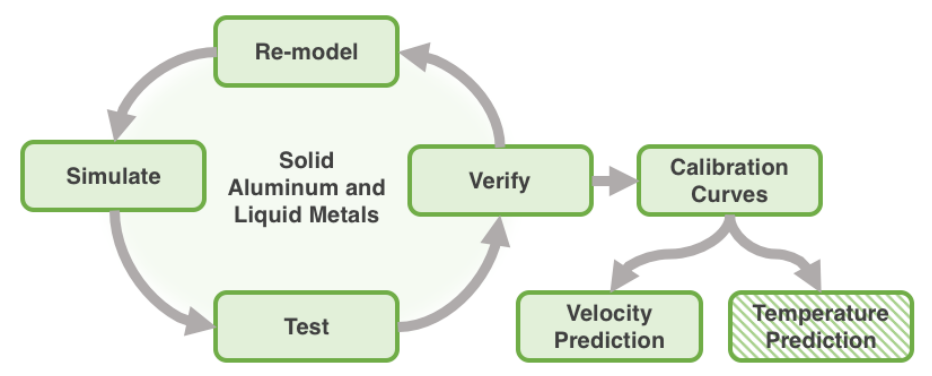

Figure 1. ECFM development process.

calibration is accurate and reliable for the dry test scenario, the experiment will proceed to the liquid testing phase.

In summary, the importance of a proper model for the ECFM is multifaceted. First, creating an experimentally verified sensor model allows for future installations of the ECFM to be easily designed and calibrated. This saves on cost and reduces installation and setup calibration times. It also enables multi-sensor use cases to be utilized in a predictable manner, such as mapping the velocity surrounding the reactor core. Additionally, once an empirical model is simulated, it can be used in more detailed system simulations of the reactor's operation, improving future studies in sensors and controls, data science, and computational fluid dynamics.

\subsection{Organization of This Report}

The report begins with the "Background" chapter, which outlines the creation of the ECFM and its basic principles. Initial prediction and analysis of the ECFM's performance is reviewed to elucidate the theory of the ECFM's behavior in "Theory of Operation." This includes some explanation of the physics which describe the phenomena being measured. Next, the placement of the ECFM is discussed in "Operational Environment." This leads to the experimental stages necessary to complete the testing, as presented in "Experimental Overview." After the foundation of the sensor properties are discussed, the "Development of the Sensor" section explains the design and build process for the ECFM. Associated requirements of operation can be reviewed in the "Sensing Requirements" section. With these requirements established, a sensor model which gives deeper insight into the device and tuning capabilities is investigated. The modeling process in COMSOL ${ }^{\mathrm{TM}}$ is then presented. The outcome of these efforts is described in the "Results" chapter within the "Numerical Simulations, "Experimental Measurements," and "Discussion" sections. Lastly, a summary of the presented results and next steps are provided in the "Conclusion." 


\section{BACKGROUND}

Figure 2 depicts the ECFM in a drawing from the Hanford Engineering and Development Laboratory(HEDL) Research and Development Transmittal(RDT) RDT C 4-7T [June 1973]. The theory to follow gives a more detailed explanation of its operation mathematically, but a short discussion of its operating concepts is provided relating to Figure 2 and 3a. As shown in Figure 2, the probe consists of a primary excitation coil (there are two in the figure, but only one is necessary), and two secondary coils. The primary coil is driven with an external current source which then produces a magnetic field surrounding the solenoid. In reaction to this introduced magnetic field, eddy currents develop in the medium which act to maintain magnetic flux density in the regions as it was prior to the excitation. This is due to Lenz's Law as well as Ampere's Law, which are explained further in the next section. Figure 3a shows the action of these laws.
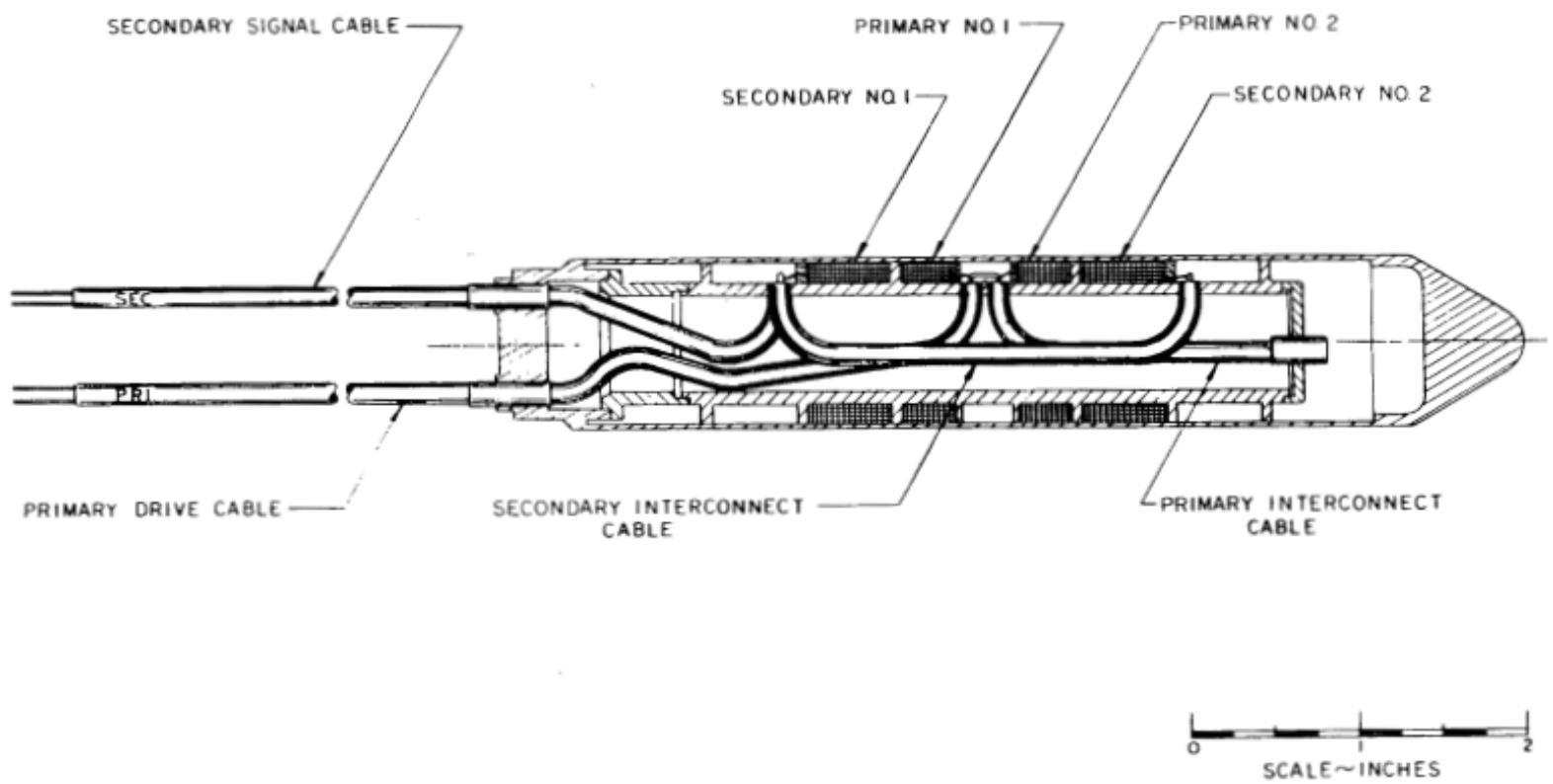

Figure 2. RDT specification ECFM probe, RDT C 4-7T [June 1973].

Wiring of the ECFM follows that of the linear variable differential transformer (LVDT) shown in Figure 3 b. The difference for the ECFM is that the medium flowing through or around the sensor is paramagnetic, whereas traditional LVDTs use an iron core to sense displacement. Either way, the principal operation of both devices is based on similar physics.

Much of the theory presented below is from Davidson [2001]. Following the discussion of the ECFM operation, the experimental history which has lead to the first prototype development will be reviewed. Operation of the ECFM will be explained through basic electromagnetic (EM) and magnetohydrodynamic (MHD) equations. The method of solving the equations is numeric, and COMSOL ${ }^{\mathrm{TM}}$ is used to complete this task. MHD investigations will be continued when the liquid testing phase becomes more complex, so 


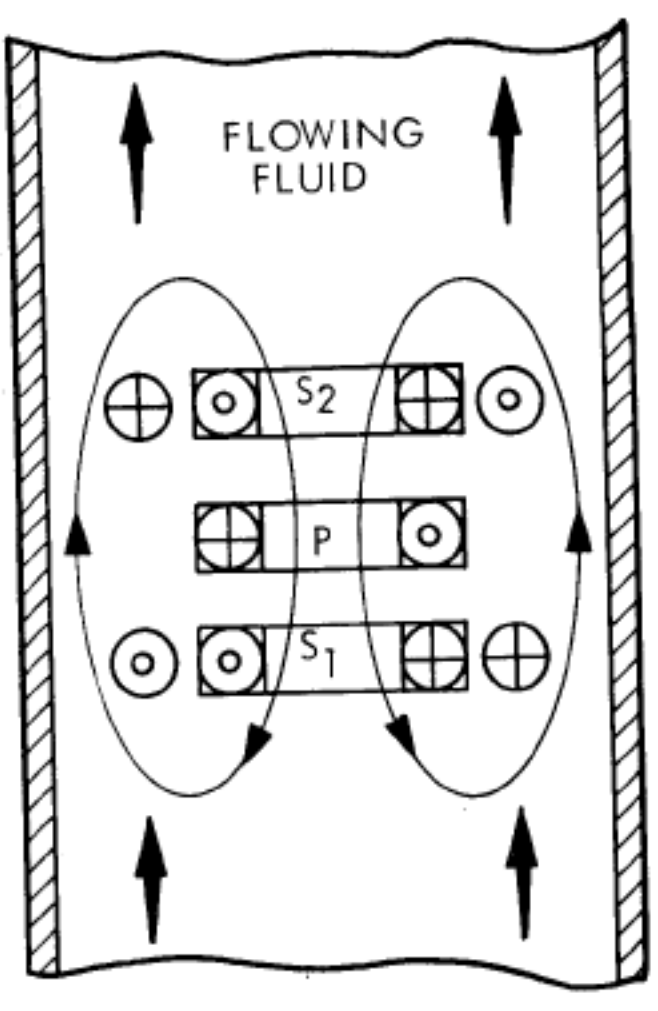

(a) RDT operation
IDEALLY BALANCED

DIFFERENTIAL TRANSFORMER

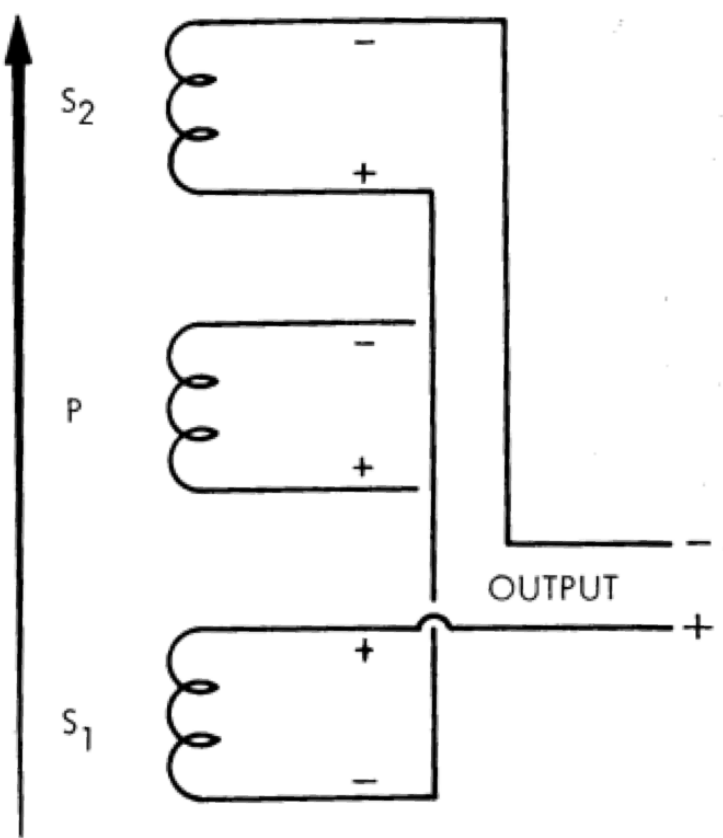

(b) ECFM wiring

Figure 3. ECFM operation and wiring, RDT C 4-7T [June 1973].

most of the theory presented is EM in nature. The result of the ECFM's operation is to produce calibration curves to be used in the field. Calibration curves corresponding to the output voltage and a known velocity are generated from modeling and lab testing. In the field they will be further verified, and the measurements will be correlated to a velocity based on the environmental conditions and sensor parameters. With the help of the experimentally verified sensor model, the process of calibration can become greatly improved. This is one of the primary goals of this study. 


\subsection{Theory of Operation}

Operation of the ECFM starts by exciting the primary coil to produce a time-varying magnetic field in a conductive medium. This is governed by Ampere's Law, which is described as

$$
\nabla \times \vec{B}=\mu\left(\vec{J}+\varepsilon_{0} \frac{\partial \vec{E}}{\partial t}\right) .
$$

The displacement current, $\varepsilon_{0} \frac{\partial \vec{E}}{\partial t}$, can be ignored in MHD since generally $\varepsilon_{0} \frac{\partial \vec{E}}{\partial t} \ll \vec{J}$. Eddy currents are induced in the medium to generate an opposing magnetic field to counteract the time varying magnetic field generated by the primary coil excitation. This effect also induces a time varying current in the secondary coils, while the magnetic flux is threading through these coils along its axial direction. The azimuthal component of the electric field is the tangential component at the boundary layer of the conductive medium, $\vec{E}_{T 1}$, and the exciting coil, $\vec{E}_{T 2}$. Setting the boundary condition $\vec{n} \times\left(\vec{E}_{T 2}-\vec{E}_{T 1}\right)=0$ implies that the tangential electric fields in the medium and that at the excited coils are continuous at the interface. Thus, an electric field is present in the secondary coils to satisfy the boundary condition; while the total tangential field at the conductor should go to zero. This also corresponds to an electromotive force (emf) and induced current. This emf is described with Faraday's Law of Induction in integral form as,

$$
\varepsilon=\oint_{C} \vec{E} \cdot d l=-\frac{d}{d t} \iint_{S} \vec{B} \cdot d \vec{S}
$$

With the secondary coils wired symmetrically and in a differential configuration as shown in Figure $3 b$, the induced voltages are equal in magnitude but opposite in phase, and they cancel. With a preliminary magnetic field established in the medium, the Lorentz force phenomenon can be measured when a flow exists which interacts with the magnetic field. The Lorentz force considering a single charged particle is expressed as

$$
\vec{f}=q(\vec{E}+\vec{v} \times \vec{B})
$$

where $q, \vec{E}$, and $\vec{B}$ are the charge of the particle, the electric field, and the magnetic flux density, respectively. From the equation, it is seen that the Lorentz force is the summation of the forces on a charged particle due to the electric field and the cross product of the particle's velocity with the magnetic field. When considering the contributions of all particles in the medium, this force is what acts on the secondary coils to induce the secondary coil currents. This is described by Ohm's law in Eq. (4) and the volumetric Lorentz Force in Eq. (5) (non-relativistic),

$$
\begin{gathered}
\vec{J}=\sigma(\vec{E}+\vec{V} \times \vec{B}), \\
\vec{F}=\vec{J} \times \vec{B},
\end{gathered}
$$

where $\vec{J}, \sigma, \vec{V}$, and $\vec{F}$ are the total induced current, electrical conductivity, flow velocity of the medium, and volumetric force. The resulting voltages in the secondary coils are measured and their magnitudes summed, resulting in the output voltage taken from the sensor. This output is proportional to the velocity of 
the medium. By combining Eq. (1), Eq. (2) in differential form, and Eq. (4), the induction equation for $\vec{B}$ is found:

$$
\frac{\partial \vec{B}}{\partial t}=\nabla \times(\vec{v} \times \vec{B})+\frac{1}{\mu \sigma} \nabla^{2} \vec{B}
$$

By replacing $\vec{B}$ with the curl of the magnetic vector potential $\nabla \times \vec{A}$ and adding the excitation source, a numerical calculation for the desired $\vec{E}$ and $\vec{B}$ fields can be performed. The time harmonic equation from manipulating Eq. (6) and adding an exciting current source due to the primary coil becomes

$$
\nabla^{2} \overrightarrow{\mathrm{A}}-\mu \sigma(\overrightarrow{\mathrm{V}} \times(\nabla \times \overrightarrow{\mathrm{A}}))+j \omega \sigma \overrightarrow{\mathrm{A}}=\mu_{0} \overrightarrow{\mathrm{J}}_{\text {exc }} .
$$

This will be performed in COMSOL ${ }^{\mathrm{TM}}$ and the results are presented in Section 4.1.

\subsection{Experimental Overview}

Choices for the ECFM experimental setup include either the probe insert or flow-through configurations, as seen in Figures $4 \mathrm{a}$ and $4 \mathrm{~b}$.

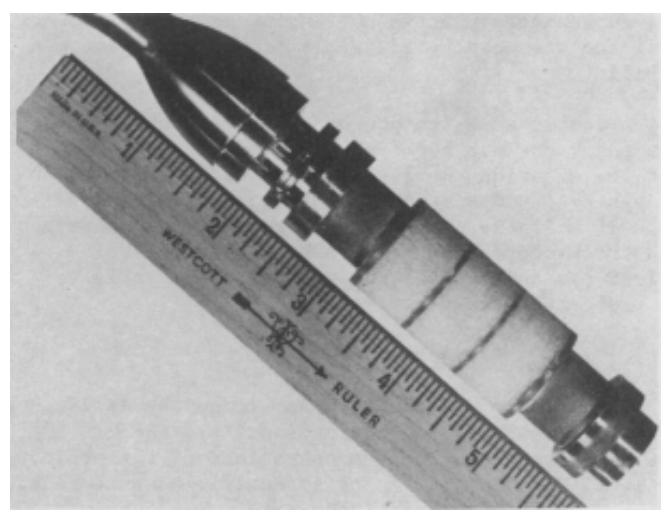

(a) Probe insert (wet case), Brewer and Jaross [1970]

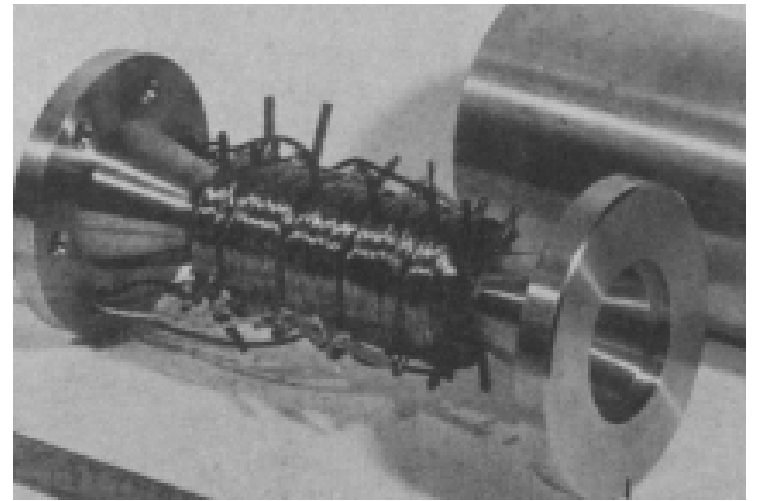

(b) Flow-through, Wiegand and Michels [1969]

Figure 4. Types of ECFMs.

The probe insert is submerged in the liquid coolant, offering the advantage of removing a boundary (the flow pipe) between the magnetic field generated from the excited coil and the conductive medium. This configuration has been tested in what are called wet and dry test setups. Wet refers to the primary and secondary coils being exposed to the liquid, although they are coated hermetically in ceramic. The $d r y$ case covers the sensor in a protective stainless steal sheath, but it results in a loss in output voltage due to the additional conductive medium between the exciting coils and the liquid. Early experiments for the probe insert were performed by John Brewer and Robert A. Jaross in 1970 at Argonne National laboratory based on an analysis of the ECFM developed by David E. Wiegand, who experimented with the flow-through type in 1969, Wiegand and Michels [1969]. More probe insert tests were performed showing the sensitivity 
degradation of the dry versus wet test case in 1973 by Westinghouse Electric Corporation, Costello et al. [1973]. Results showed more than a 50\% decrease in the average recorded output voltage from the sensor in the dry test case. An example of the dry test case sensor is shown in Figure 5a, and the dry versus wet test case voltage comparison is shown in Figure 5b. The decrease in output voltage can be accommodated for with further knowledge of the sensor parameter relations.

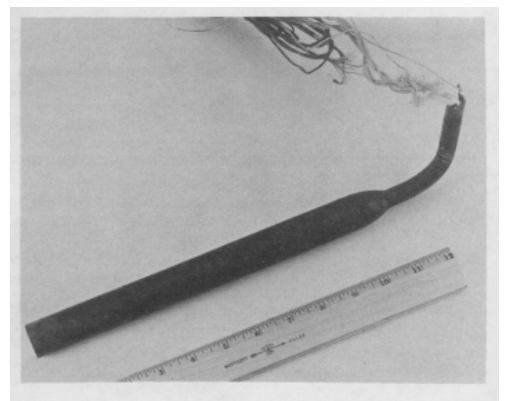

(a) Dry test probe example inside a SS Sheath, Brewer and Jaross [1970]

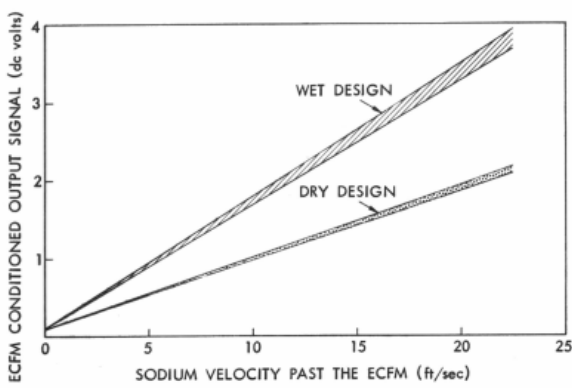

(b) Comparison of wet vs. dry test case sensor output, Costello et al. [1973]

Figure 5. Types of ECFMs (continued)

Alternatively, flow-through sensor configurations offer the advantage of easy attachment to a flow channel and an increased magnetic field intensity in the liquid flow. This is because the maximum magnetic field intensities take place inside of the solenoid, although the addition of the pipe channel and its material properties can counteract some of the increased sensor output for the flow-through ECFM. In terms of its convenience for prototyping and mounting, the flow-through ECFM was chosen for the initial experiments to understand the ECFM operation principles and to verify the effects of the sensor parameters on output voltage, calibration, and performance. Probe insert experiments require special material coating of the sensor coils, mineral insulated cabling, and mounting of the sensor inside of the flow channel. This would make characterization and sensor modification highly inefficient for the initial ECFM investigation. Since calibration is sensor-dependent and environmentally dependent, it is believed that the flow-through prototype will provide the necessary foundation for designing both a flow-through and a probe insert ECFM. A comparison of some original experimental results from the probe insert and flow-through prototypes are shown in Figures 6a and 6b. The comparison is not direct, but it does show the relationships of sensor output voltage and flow velocity, as well as sensor output voltage and frequency, that are expected. Both experiments provide value in benchmarking future simulations and ECFM sensor prototypes when the project continues to the liquid testing phase. Figure 6a shows the linear sensor output voltage vs. flow velocity. Figure $6 \mathrm{~b}$ shows the multi-valued and exponentially decaying sensor output voltage vs. frequency. The $y$-axis in Figure $6 \mathrm{~b}$ is the ratio of the sensor output voltage to the flow velocity and is defined as sensitivity. Each relationship has been modeled with a new sensor prototype and confirmed in simulation. The prototype and results are presented in Section 4.1. 


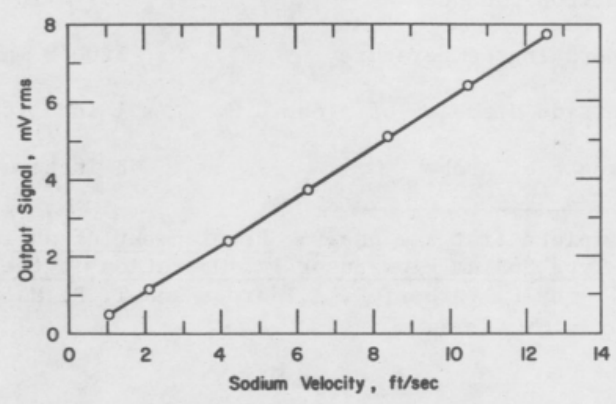

(a) Probe insert experiment: sensor output vs. flow velocity, Brewer and Jaross [1970]

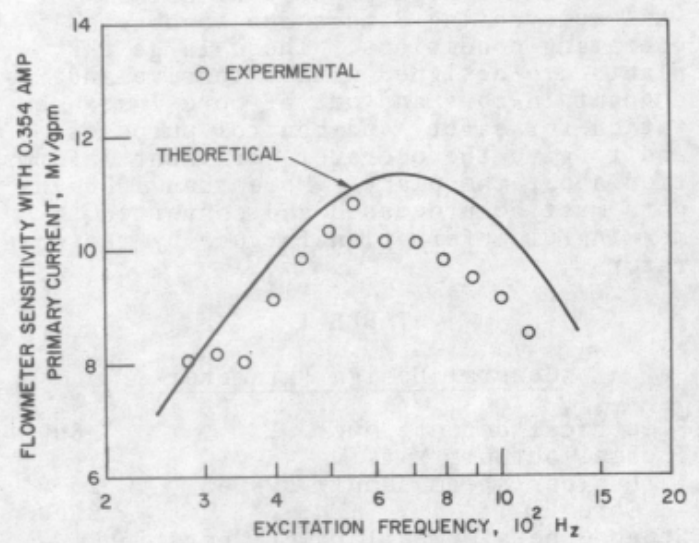

(b) Flow-through experiment: sensor output/flow rate vs. quency, Wiegand and Michels [1969]

Figure 6. Sensor output relations

\subsection{Operational Environment}

The ECFM was originally a part of the Fast Flux Test Facility (FFTF) measurement systems as shown by Nutt and Stringer [March 1973]. Previous locations of the ECFM near the reactor core at the FFTF are shown in Figure 7. The figure shows the possibilities for velocity, temperature, and void mapping of the core using multiple ECFMs. A more structural graphic of the ECFM location is shown in Figure 8a, where the ECFM is located in the Fuels Open Test Assembly (FOTA) instrument stalks above the core. An internal look at the location of the ECFM in the FOTA is shown in Figure 8b.

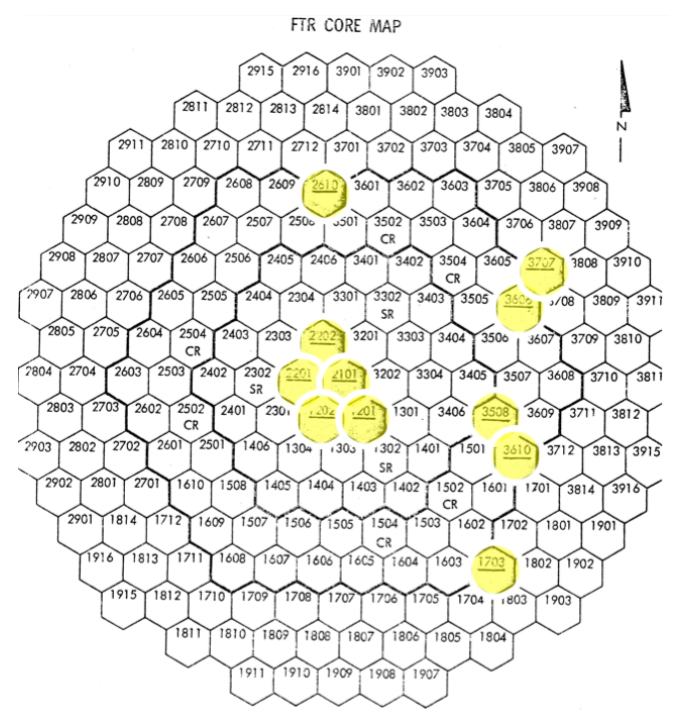

Figure 7. ECFM core placement, Nutt and Stringer [March 1973]. 


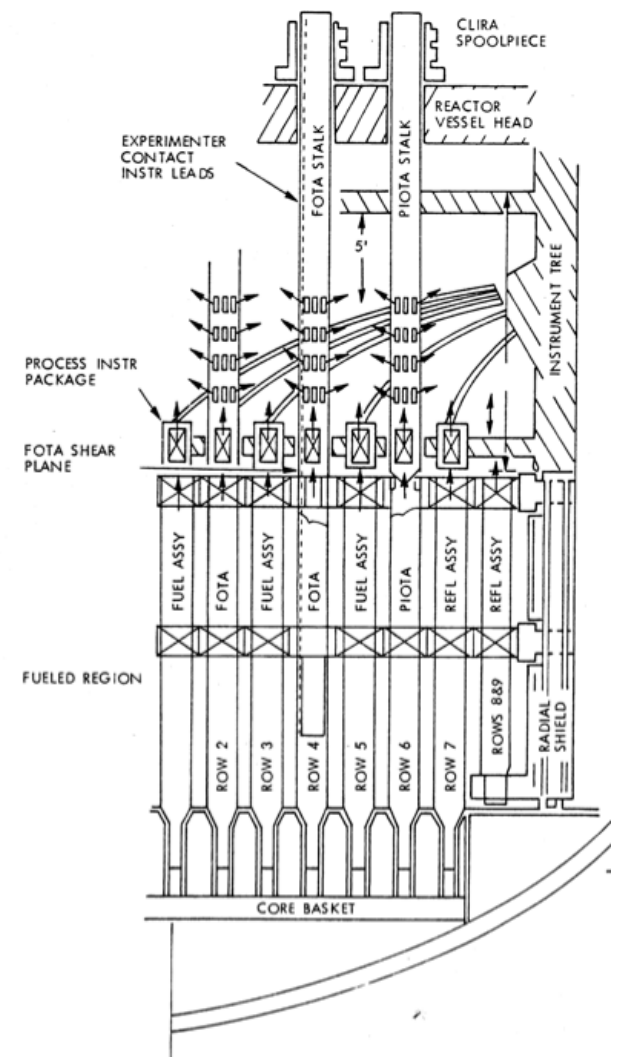

(a)

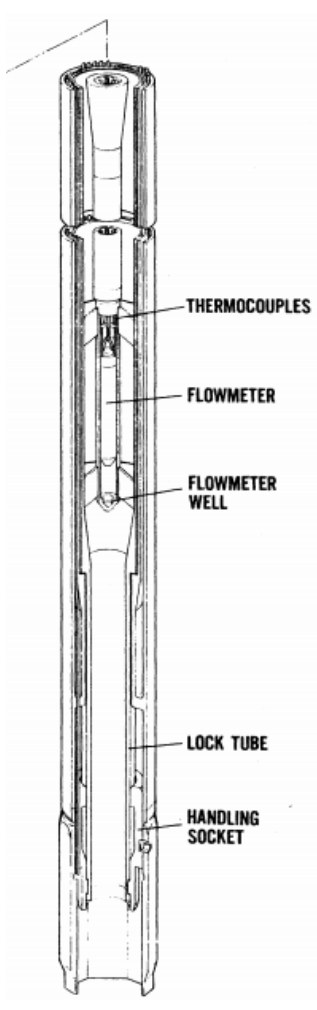

(b)

Figure 8. FOTA and ECFM placement, Nutt and Stringer [March 1973].

\subsection{Aluminum Rod Testing}

The initial experimental setup for the ECFM can be seen in Figure 9a, and the sensor is shown in Figure 9b.

It consists of the sensor with a 6061-T6 aluminum rod as the conductive medium. The sensor prototype is wound with 26 AWG ( $0.405 \mathrm{~mm}$ diameter) copper magnet wire. There are 70 primary turns, and the section is $30 \mathrm{~mm}$ long. Both secondary sections have 50 turns each and are $20 \mathrm{~mm}$ long. Coils are wound on a delrin (polyoxymethylene) bobbin. The sensor is mounted on a motion track capable of speeds up to $2.5 \mathrm{~m} / \mathrm{s}$. Track motion is facilitated with a linear belt drive actuator and is controlled by a Kinco FD422-LA-000 servo drive using incremental encoding. A position loop has been set on the driver to move the sensor on the aluminum rod. Driving the device is a Kepco BOP (Bipolar Operational Amplifier) 20-5DL optimized for inductive loads. Its current programming mode is used to amplify a signal generator output from a NI PXI-5421 to produce a constant current excitation to the sensor's primary coils. A Stanford Research 850(SR850) lock-in amplifier is used as a differential amplifier to measure the secondary coil difference and can also be used as the driving signal. The lock-in amplifier also serves the purpose of phase sensitive detection to increase the signal-to-noise ratio (SNR) of the sensor output measurement by reducing the bandwidth of the measured signal. The NI PXI-5421 programming signal is also sent to the external frequency reference of the lock-in amplifier to perform phase-sensitive detection if the internal reference on the lock-in is not used. Voltage differences in the range of $2 \mathrm{nV}$ to $1 \mathrm{~V}$ can be 


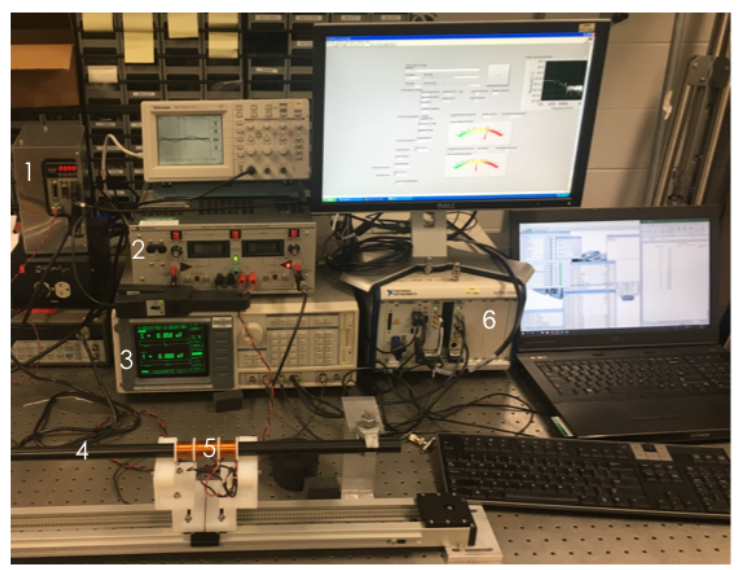

1.Servo Controller

2.Excitation Signal

3.Lock-in Amp

4.Al 6061-T6 Rod (Black)

5.Sensor

6.LabVIEW (Hardware)

(a)

Figure 9. Experimental setup (a) and prototype sensor (b).

measured in this configuration with proper filter settings. Finally, the SR850 output is fed to the PXI-5922 16 bit digitizer and processed in LabVIEW. Based on the sensitivity set on the SR850, the output scaling behaves as follows,

$$
V_{\text {scaled }}=\left(\frac{V_{\text {sensor }}}{V_{\text {sensitivity }}}-D C_{\text {offset }} \%\right) \cdot \text { expand } \cdot 10
$$

where $V_{\text {scaled }}$ is the measured output signal from the lock-in amplifier, $V_{\text {sensor }}$ is the sensor output signal fed to the lock-in, and $V_{\text {sensitivity }}$ is the voltage sensitivity selected for the measurement. $D C_{\text {offset }} \%$ is added if the lock-in output signal is set to a $0 \mathrm{~V}$ reference. Expand increases the resolution of the output signal if needed. 


\section{DEVELOPMENT OF SENSOR}

To develop the sensor, it was decided to first model simulation results from previous literature for the ECFM to gain a better understanding of the sensor's behavior. Doing this allows the first prototype built to be based on prior simulations so that a benchmark for the model can be obtained. Then further model and experimental verification can be performed. The clearest ECFM model found in the literature is from Kumar [2016], and the same results have been replicated. This has formed the basis of the prototype presented in Section 4.1. The prototype is a flow-through sensor, which, as discussed previously, allows for producing a simple testable sensor. However, the final probe will be a probe-type ECFM. Complexity will advance to reach the original RDT C 4-7T standards presented in RDT C 4-7T [June 1973] for an ECFM probe. The RDT C 4-7T specification gives material properties, fabrication, and signal requirements for developing the ECFM. It is believed to be based on the sensor presented in Brewer and Jaross [1970], which was developed for the FFTF in 1970 at Argonne National Laboratory. Progress made so far with the flow-through type sensor will greatly aid in the final construction of the probe-type sensor defined by the RDT specifications.

\subsection{Sensing Requirements}

The probe is shown again in Figure 10 for convenience. Table 1 shows the materials listed in the RDT specification. There is a note which states that non-proprietary materials (those not the same as what is listed in the specification) must meet or exceed the performance of the listed materials. This will be helpful if more modern materials can be used to replace that recommended in the specification. SS refers to Stainless Steel. The coils are wet wound in ceramic cement and are also coated with Alumina plasma arc spray. Mineral-insulated cables provide the path for the sensor excitation and output from the system. These cables are chosen to withstand the harsh nuclear environment and operate on the principle of thermocoupling. Thermocouples convert temperature changes from two different wire materials into a measurable voltage. Lead wires are further supported with stainless steel mounts at the end of the sensor where the mineral-insulated cables enter. A gas fills the cable run with helium and is finally surrounded by a thimble or sensor sheath. If the wet design is chosen, there is no sensor sheath as illustrated in the wet vs. dry sensor results presented above. The following tables are reproduced from the RDT C 4-7T Standard developed in 1973 at the Hanford Engineering Development Laboratory, RDT C 4-7T [June 1973]. 


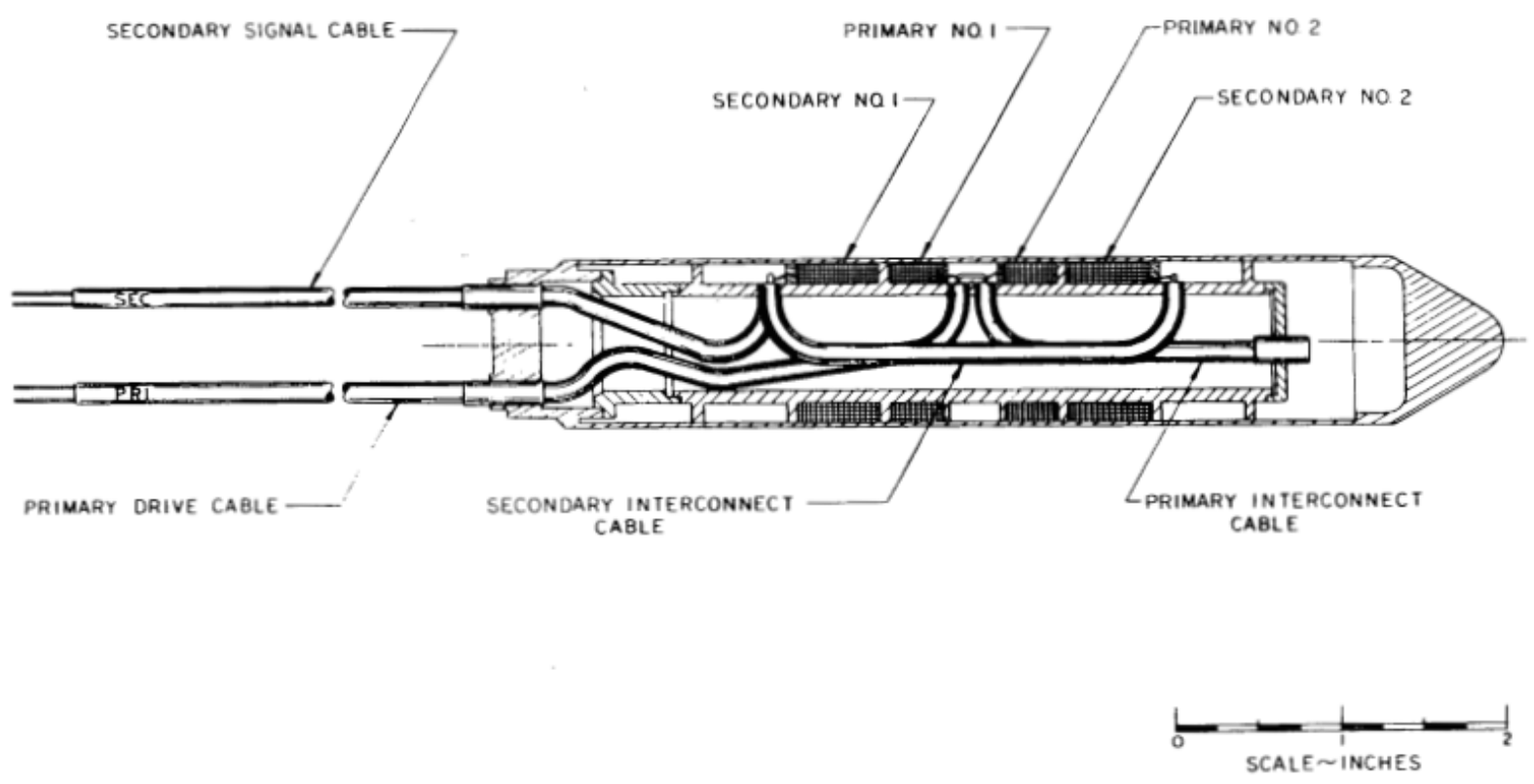

Figure 10. RDT specification ECFM probe, RDT C 4-7T [June 1973].

Table 1. RDT material specifications

\begin{tabular}{|c|c|c|}
\hline Sensor part & Material & Additional Specifications \\
\hline Nose piece & 304 SS 1.125 in. & RDT M 7-3 \\
\hline Lead wire support & 304 SS tube 0.187 in. OD ×0.042 in. minimum wall & RDT M 3-2 \\
\hline Sensor sheath & 304L SS pipe 3/4 IPS Schedule 10 & RDT M 3-6 \\
\hline Coil bobbin & Ni-Cr-Mo-Co Alloy 1 in. OD bar & ASTM B466 \\
\hline Coil wire & Gold wire & Described in literature \\
\hline Coil cement & Ceramic & Described in literature \\
\hline Wire insulation & Ceramic & RDT C 18-1 \\
\hline Fill gas & Helium & 99.995\% pure with 5 ppm water maximum \\
\hline Plasma arc spray & Alumina & Rold \\
\hline
\end{tabular}

The fill gas in Table 1 refers to the insulating gas surrounding the probe insert. An example is shown in Figure 11. Requirements for the output signal, quality testing, and fabrication are also listed in the table. Fabrication requirements clarify the welding and testing requirements for constructing the sensor. Other signal and performance requirements can be seen in Table 2 . 


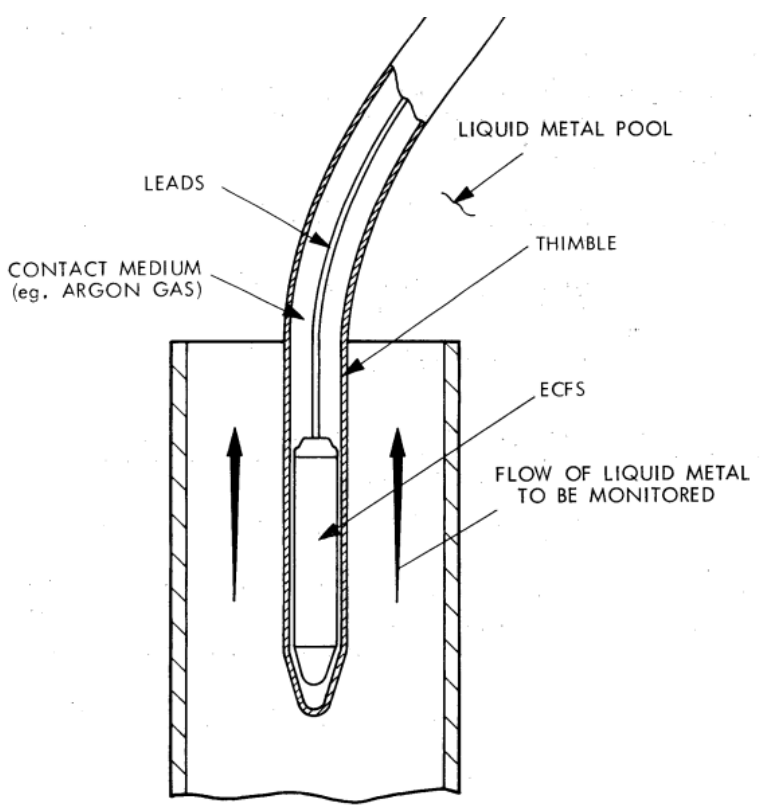

Figure 11. Probe example with fill gas, RDT C 4-7T [June 1973].

Table 2. RDT performance requirements

\begin{tabular}{|l|l|}
\hline Parameters & Requirement \\
\hline Velocity range & $0-7.6 \mathrm{~m} / \mathrm{s}$ \\
\hline Repeatability & Sensor output must be repeatable withing $\pm 3 \%$ of full range \\
\hline Accuracy & Within $10 \%$ of full range \\
\hline Response time & $63 \%$ response to a step change is to be less than $50 \mathrm{~ms}$ \\
\hline Signal output & Sensitivity is required to exceed $0.75 \mathrm{mv} / \mathrm{m} / \mathrm{s}$ \\
\hline Service life & Must operate in a specified environment for a minimum of 3 years \\
\hline Zero flow signal balance & $\begin{array}{l}\text { Output must be less than } 1 \mathrm{mV} \mathrm{rms} \mathrm{under} \mathrm{zero} \mathrm{flow} \mathrm{conditions} \mathrm{and} \\
\text { of the same phase as the input signal }\end{array}$ \\
\hline Environmental requirements & To be discussed when temperature testing phase begins \\
\hline Radiation requirements & To be discussed when radiation testing begins \\
\hline
\end{tabular}

The previous sections outline this project's final product. The results for the initial prototype modeling that has been completed are provided below. 


\section{RESULTS}

The goal of the prototype is to verify the sensor parameter relations and significance derived from in-depth simulations. When the simplest simulation and prototype are verified experimentally, more complicated simulations involving additional sensor output dependencies will be investigated.

\subsection{Numerical Simulations: Aluminum Rod}

Figure 12 shows the sensor prototype which was modeled in $\mathrm{COMSOL}^{\mathrm{TM}}$ using a $2 \mathrm{D}$ axisymmetric model. The bobbin design is shown in Figure 13. The model has been modified to 3D to illustrate its geometry more clearly. Modeling includes the delrin bobbin, magnet wire copper coils, and a 6061T aluminum rod.

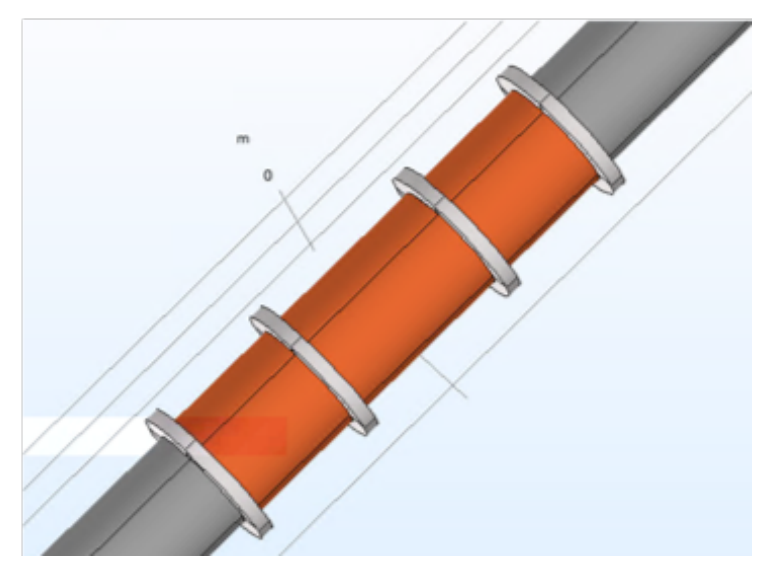

Figure 12. Sensor prototype model.

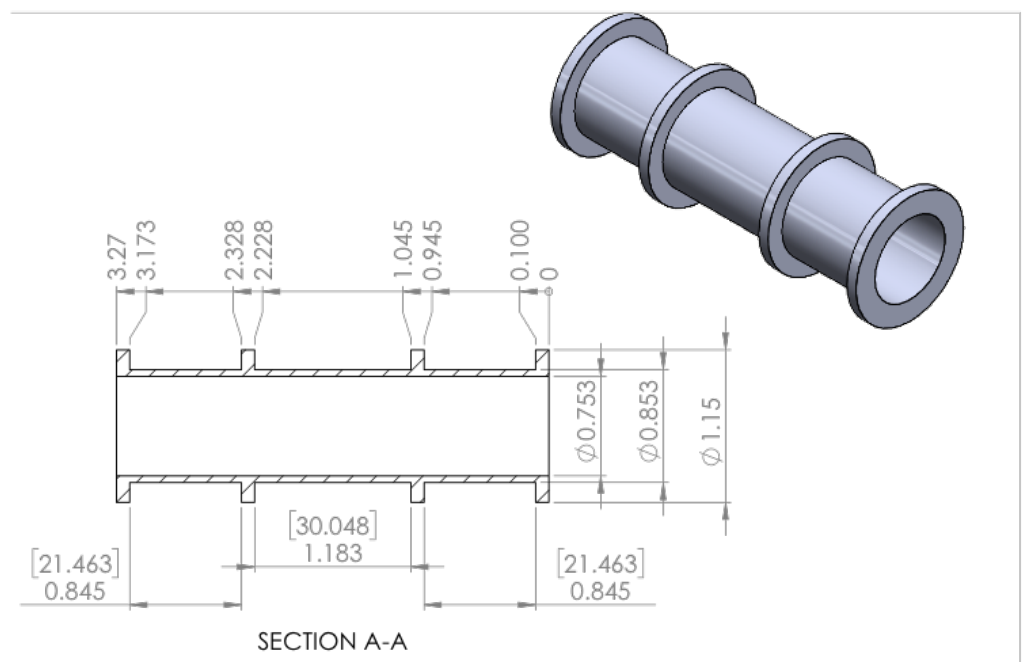

Figure 13. Bobbin design. 
Prototype parameters are shown in Table 3. Material properties for the copper coils, aluminum 6061-T6 rod, and delrin bobbin are shown in Table 4.

Table 3. First prototype sensor parameters

\begin{tabular}{|c|c|}
\hline Parameters & Values \\
\hline Primary turns & 70 \\
\hline Primary length & $30 \mathrm{~mm}$ \\
\hline Secondary turns & 50 \\
\hline Secondary length & $21.46 \mathrm{~mm}$ \\
\hline Sensor diameter & $19.13 \mathrm{~mm}$ \\
\hline Coil gauge & 26 AWG $(0.406 \mathrm{~mm})$ \\
\hline Bobbin thickness & $2.54 \mathrm{~mm}$ \\
\hline Coil spacing & $2.54 \mathrm{~mm}$ \\
\hline
\end{tabular}

Table 4. Sensor material properties (@293 K)

(a) Copper simulation properties

\begin{tabular}{|c|c|}
\hline Parameter & Value \\
\hline Density & $8,960 \frac{\mathrm{kg}}{\mathrm{m}^{3}}$ \\
\hline$\varepsilon_{r}$ & 1 \\
\hline$\mu_{r}$ & 1 \\
\hline$\sigma$ & $5.998 E^{7} \frac{\mathrm{S}}{\mathrm{m}}$ \\
\hline Heat capacity & $385 \frac{\mathrm{J}}{\mathrm{kg} \cdot \mathrm{K}}$ \\
\hline Thermal conductivity & $400 \frac{\mathrm{W}}{\mathrm{m} \cdot K}$ \\
\hline Coefficient of thermal expansion & $16 E^{-} 6 \frac{1}{K}$ \\
\hline Young's modulus & $110 E^{9} \mathrm{~Pa}$ \\
\hline Poisson's ratio & 0.35 \\
\hline
\end{tabular}

(b) Aluminum 6061-T6 simulation properties

\begin{tabular}{|c|c|}
\hline Parameter & Value \\
\hline Density & $2,700 \frac{\mathrm{kg}}{\mathrm{m}^{3}}$ \\
\hline$\varepsilon_{r}$ & 1 \\
\hline$\mu_{r}$ & 1 \\
\hline$\sigma$ & $2.46 E^{7} \frac{\mathrm{s}}{\mathrm{m}}$ \\
\hline Heat capacity & $900 \frac{\mathrm{Jg}}{\mathrm{kg} \cdot \mathrm{K}}$ \\
\hline Thermal conductivity & $238 \frac{\mathrm{W}}{\mathrm{m} \cdot \mathrm{K}}$ \\
\hline Coefficient of thermal expansion & $23 E^{-} 6 \frac{1}{K}$ \\
\hline Young's modulus & $70 E^{9} \mathrm{~Pa}$ \\
\hline Poisson's ratio & 0.33 \\
\hline
\end{tabular}

(c) Delrin simulation properties

\begin{tabular}{|c|c|}
\hline Parameter & Value \\
\hline Density & $1,420 \frac{\mathrm{kg}}{\mathrm{m}^{3}}$ \\
\hline$\varepsilon_{r}$ & 3.7 \\
\hline$\mu_{r}$ & 1 \\
\hline$\sigma$ & $1 E^{-} 15 \frac{\mathrm{S}}{\mathrm{m}}$ \\
\hline Heat capacity & $385 \frac{\mathrm{J}}{\mathrm{kg} \cdot K}$ \\
\hline Thermal conductivity & $0.36 \frac{\mathrm{W}}{\mathrm{m} \cdot K}$ \\
\hline Coefficient of thermal expansion & $16 E^{-} 6 \frac{1}{K}$ \\
\hline Young's modulus & $2.9 E^{9} \mathrm{~Pa}$ \\
\hline Poisson's ratio & 0.37 \\
\hline
\end{tabular}

Coils were modeled using the AC/DC Module's built in multi-turn coil objects. The motion of the aluminum rod was modeled with the Solid Mechanics Module. The choice of rod movement in simulation 
vs. sensor movement in the lab setup should have no discrepancies, as the reference frame does not alter the measured phenomena. Sensor movement was chosen in the lab for safety and spatial concerns. Figure 14a describes the sensor output (sensitivity*) and rod velocity relation. For a given excitation signal, the sensitivity is clearly linear, making calibration relatively simple. However, Figure $14 \mathrm{~b}$ appears to indicate non-linearities in terms of frequency response and sensitivity. The sensitivity vs. velocity curve for frequencies below $500 \mathrm{~Hz}$ no longer follows the predicted scaling ratio between successive excitation frequencies $(250 \mathrm{~Hz}$ sensitivity is less than $500 \mathrm{~Hz}$ ). This is clearly shown in Figure 14b. Below $400 \mathrm{~Hz}$, sensitivity levels off and then decreases, so the Sensitivity vs. Frequency graph is multi-valued. These graphs indicate the ideal excitation frequencies based on the sensor geometry or vice versa.

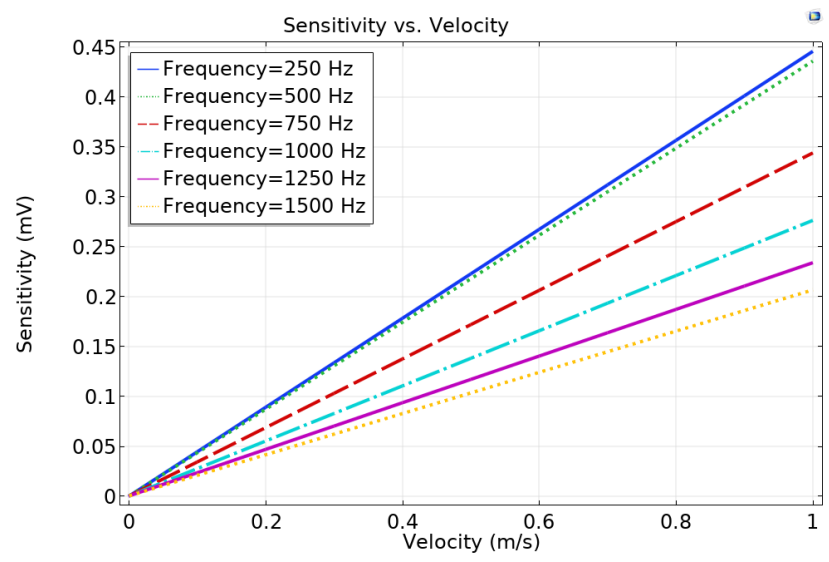

(a) Sensitivity vs. velocity

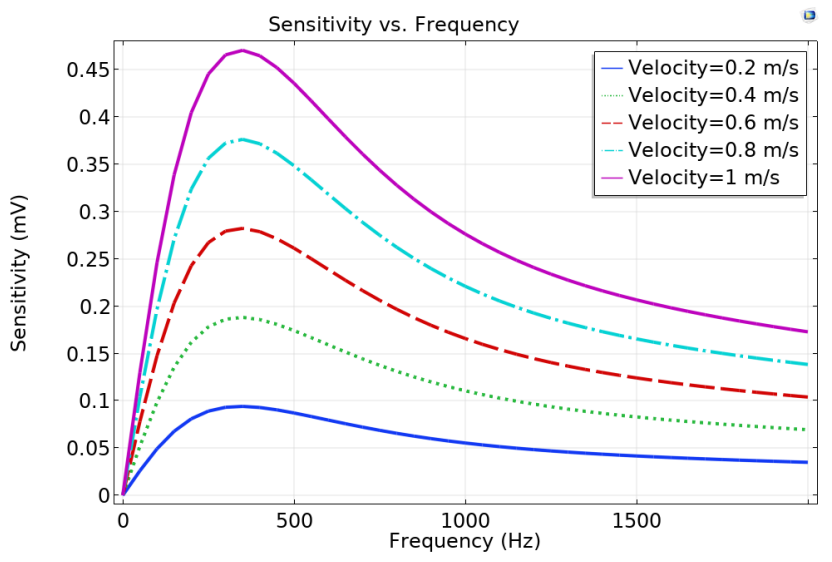

(b) Sensitivity vs. frequency

Figure 14. Sensitivity curves for $6061 \mathrm{~T}$ aluminum rod.

The maximum sensitivity level for the prototype is about $350 \mathrm{~Hz}$ considering a fixed velocity. The reason for this change in frequency response is the skin depth of the conductive medium and the diameter of the flow channel and sensor. The skin depth for a good conductor with relative permeability $\mu_{r} \approx 1$, ignoring temperature dependence on conductivity, is,

$$
\delta=\sqrt{\frac{\sigma}{\pi f \mu_{0}}}
$$

where $\sigma$ is electrical conductivity, $\mu$ is permeability of free space, and $f$ is frequency. Figure 15 shows the effect of changing the flow channel and the corresponding sensor radius. For each frequency there is a region of greatest positive increase. This occurs at a pipe radius between 1-2 skin depths for a given frequency as shown by Kumar [2016].

* Sensitivity is actually defined as the ratio of sensor output voltage to the flow rate; however, the aluminum rod does not have a flow rate. It was decided to name the measurement sensitivity for the aluminum rod testing for consistency with future liquid tests which will report both sensor output voltage and sensitivity. 


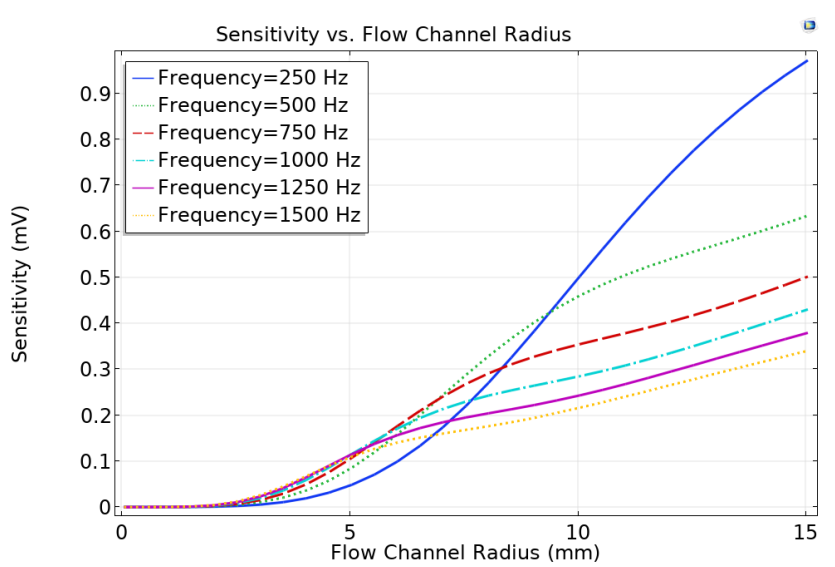

Figure 15. Sensitivity from changing flow channel radius.

This shows that the excitation frequency or frequencies used should be such that the pipe diameter is at least two times greater than the skin depth of the excitation frequency so that more of the magnetic flux density is contained in the flow channel medium.

Further simulation of the sensor shows the relationship regarding the primary and secondary coil lengths when holding the number of turns constant. This is done by stacking the coil turns or changing the coil gauge. Figures 16a and 16b show that decreasing the coil sections provides an exponential increase in sensitivity. Figure 16 verifies this relation by multiplying the turns ratio in each of the sensors sections by a turns ratio factor. Sensitivity can be greatly increased to meet a sensor's output voltage requirement by symmetrically stacking the coil turns. Given some geometric constraints on the sensor, the results can be used to determine an optimal and physically realizable set of sensor parameters. Furthermore, output sensitivity can be adjusted to combat system noise and the desired range of velocity flow or temperature.

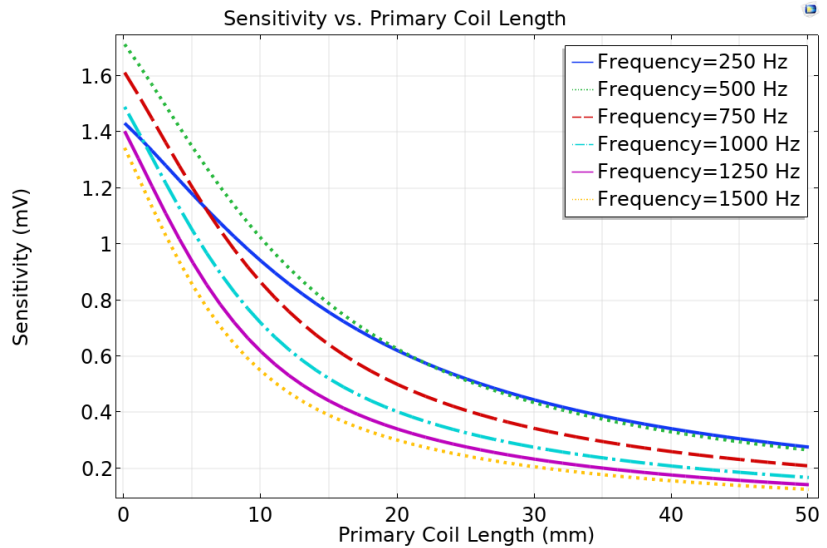

(a)



(b)

Figure 16. Sensitivity vs. coil length. 


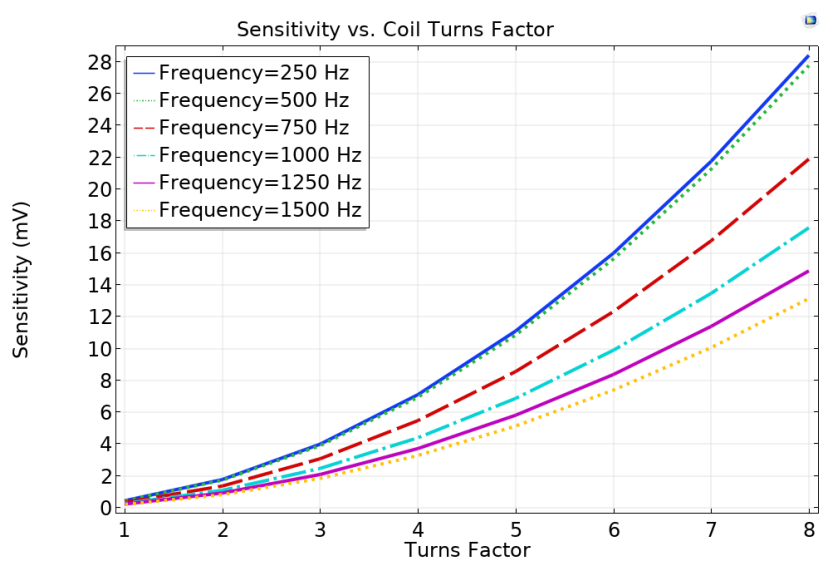

Figure 17. Sensitivity vs. coil turns ratio.

To further understand the magnetic field interaction with the moving conductive medium, a sequence of 2D magnetic flux plots in $\mathrm{COMSOL}^{\mathrm{TM}}$ were generated. Figure 18 contains a surface gradient which corresponds to the $B_{z}(r, z)$ component of the magnetic flux density, and it also includes contour lines representing the $B_{r}(r, z)$ component of the magnetic flux density. Contour lines for $B_{r}(r, z)$ tend to a value of 0 at the outer layer of the contour. The purpose of the graphics are to show that the radial component of the magnetic flux density penetrates less into the conductive medium as frequency increases. Also, the magnetic flux density decays exponentially for all frequencies when moving radially inward in the conductive medium. This explains the physical meaning of the curve in Figure 14b. According to Eq. (5), $B_{r}(r, z)$ contributes to the Lorentz force, and $B_{z}(r, z)$ does not. $B_{r}(r, z)$ is due to the fringing fields at the boundary of the primary and secondary coils, is weaker, and has less depth into the medium than $B_{z}(r, z)$. This explains the decrease in sensor output voltage seen in Figure 14b. However, the significant decrease in sensor output voltage below about $350 \mathrm{~Hz}$ can be explained by the magnetic flux density for some lower frequencies being nearly equivalent to those caused by higher frequency excitations. This occurs when the magnetic field exits the medium, thus the magnetic flux density is lower. When the radius of the flow channel is smaller than $2 \delta$ (skin depths), then the effect is significant. Although the $B_{z}(r, z)$ component does not interact with $\vec{v}$ (considering a unidirectional flow), it provides a conceptual view of the magnetic flux density exiting the medium. The contribution of $B_{z}(r, z)$ will play a significant role when vorticity of a liquid metal coolant is introduced by turbulent flow or when discontinuities caused by the placement of a probe-type ECFM affect the fluid motion. Evaluation of the probe-type ECFM will be much more complicated and less predictable in those cases. 


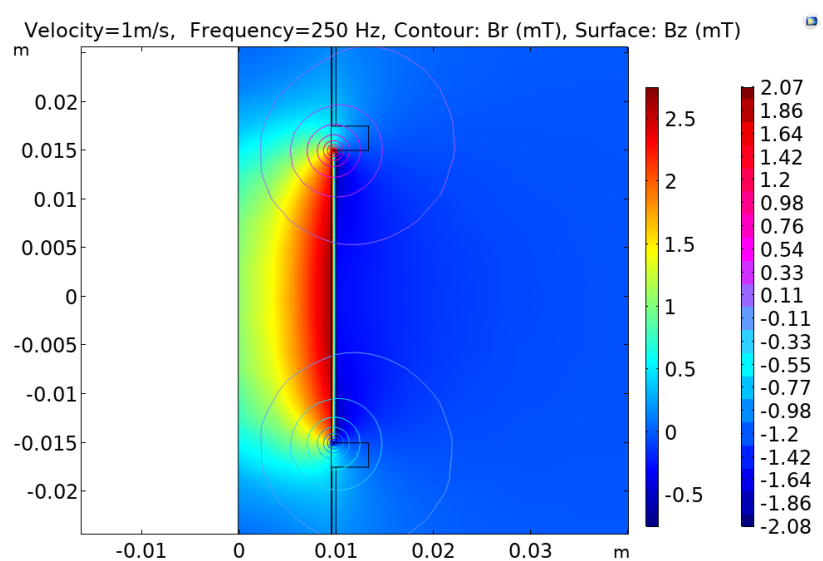

(a)

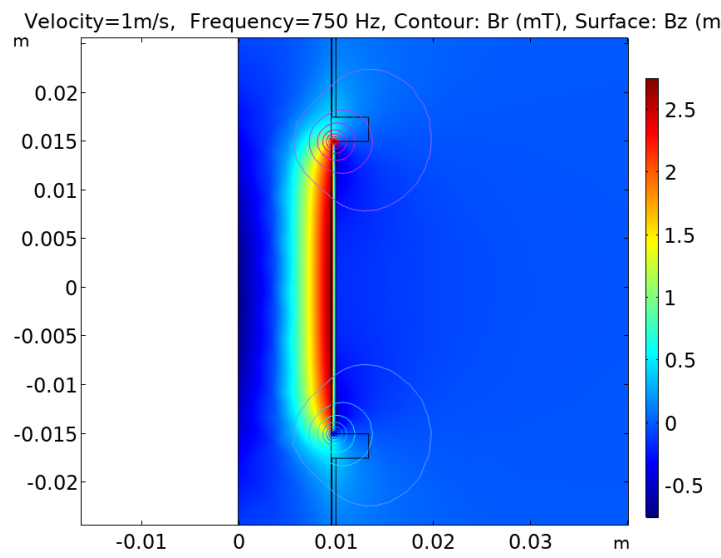

(c)

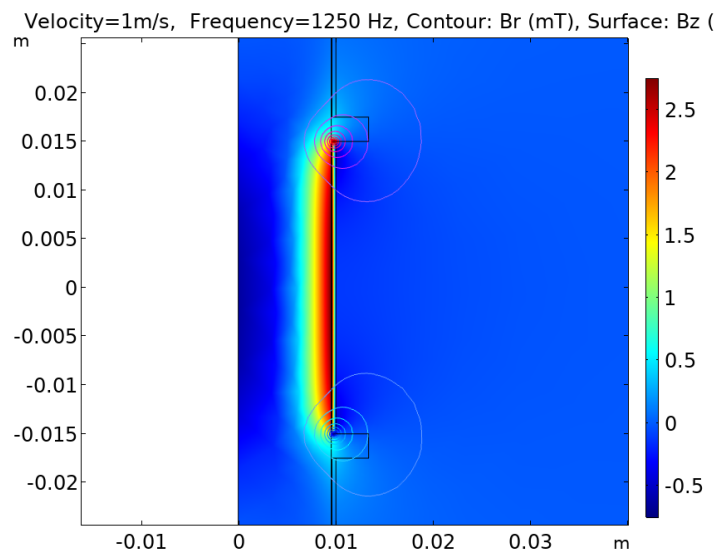

(e)

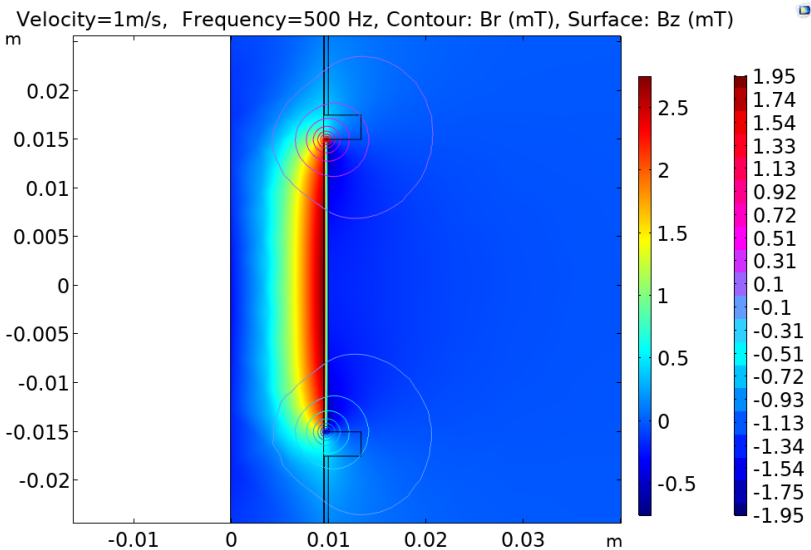

(b)

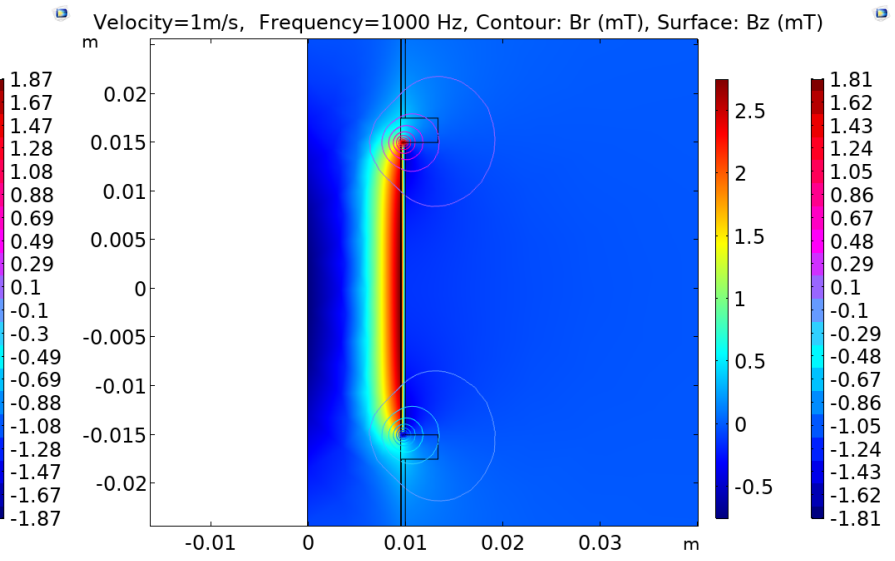

(d)

- Velocity $=1 \mathrm{~m} / \mathrm{s}$, Frequency $=1500 \mathrm{~Hz}$, Contour: $\mathrm{Br}(\mathrm{mT})$, Surface: $\mathrm{Bz}(\mathrm{mT})$ o

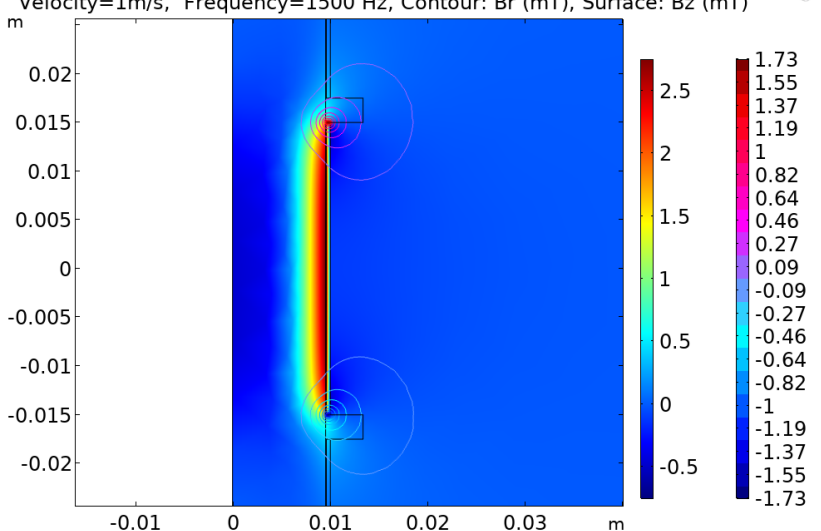

(f)

Figure 18. Magnetic flux density for $B_{z}(r, z)$ and $B_{r}(r, z)$. 


\subsection{Numerical Simulations: Mercury and Galinstan}

A comparison of a mercury and galinstan medium for the ECFM was simulated to improve understanding of the following effects:

- Viscosity effect on the fluid profile

- Liquid conductivity relation to frequency response

- Excitation frequency for the greatest sensor output voltage

- Differences in material properties on sensor behavior

Two generic liquid metals were used for the comparison. Their properties can be seen in Table 5 . Temperature dependence has not been evaluated. Material property temperature dependence curves will be extremely sensitive to the exact liquid metal to be used for experimentation. If such dependencies for temperature already exist, then the characterization of the ECFM performance will be simplified. However, it is expected that some experimental material properties will have to be determined to produce an accurate temperature model for the ECFM. Mercury was chosen because of the accessibility of the material in a liquid loop at ORNL. Galinstan was chosen because its conductivity is quite similar to that of liquid sodium. However, the effect of viscosity on the fluid profile will make galinstan and sodium results quite different when future experiments are performed. Also, the pipe dimension and therefore sensor properties will be different when experiments are conducted. The idea is to show the general operation of the ECFM in contact with the liquid metals.

Table 5. Liquid metal properties (@293K)

(a) Galinstan simulation properties

\begin{tabular}{|c|c|}
\hline Parameter & Value \\
\hline Dynamic viscosity & $0.0024 \mathrm{~Pa} \cdot \mathrm{s}$ \\
\hline Density & $0.00644 \frac{\mathrm{kg}}{\mathrm{m}^{3}}$ \\
\hline$\varepsilon_{r}$ & 1 \\
\hline$\mu_{r}$ & 1 \\
\hline$\sigma$ & $3.46 E^{6} \frac{\mathrm{s}}{\mathrm{m}}$ \\
\hline Heat capacity & $296 \frac{\mathrm{g} \cdot \mathrm{K}}{\mathrm{kg}}$ \\
\hline Thermal conductivity & $16.5 \frac{\mathrm{W}}{\mathrm{m} \cdot \mathrm{K}}$ \\
\hline
\end{tabular}

(b) Mercury simulation properties

\begin{tabular}{|c|c|}
\hline Parameter & Value \\
\hline Dynamic viscosity & $0.00155 \mathrm{~Pa} \cdot \mathrm{s}$ \\
\hline Density & $13555 \frac{\mathrm{kg}}{\mathrm{m}^{3}}$ \\
\hline$\varepsilon_{r}$ & 1 \\
\hline$\mu_{r}$ & 1.00074 \\
\hline$\sigma$ & $1.09 E^{6} \frac{\mathrm{S}}{\mathrm{m}}$ \\
\hline Heat capacity & $139.25 \frac{\mathrm{J}}{\mathrm{kg} \cdot \mathrm{K}}$ \\
\hline Thermal conductivity & $8.75 \frac{\mathrm{W}}{\mathrm{m} \cdot \mathrm{K}}$ \\
\hline
\end{tabular}

Simulation was performed with a pipe length that was five times the pipe's diameter to ensure that a fully developed laminar flow was established. The inlet of the flow channel was assigned a uniform velocity which was stepped through velocities of $0.1-1.1 \frac{\mathrm{m}}{\mathrm{s}}$.

Before presenting the velocity calibration curves, it is important to discuss the effects of the fluid profile, the material characteristics, and the significance of the excitation frequency related to those effects. The fluid profile has a direct effect on the sensor output voltage reading. As shown in Figure 19, velocity gradients extending radially from the center of the flow channel are quite different. This is believed to be a consequence of the material viscosity. The results report the mean flow velocity given by COMSOL TM considering the interaction of $\vec{V}$ and $B_{r}(r, z)$. The velocity decreases toward the wall of the flow channel, 
much more so for galinstan. Furthermore, the flow velocity near the center of the pipe will often be greater than the inlet velocity, assuming a uniform inlet flow. This effect results in an inaccurate picture of the overall flow velocity.

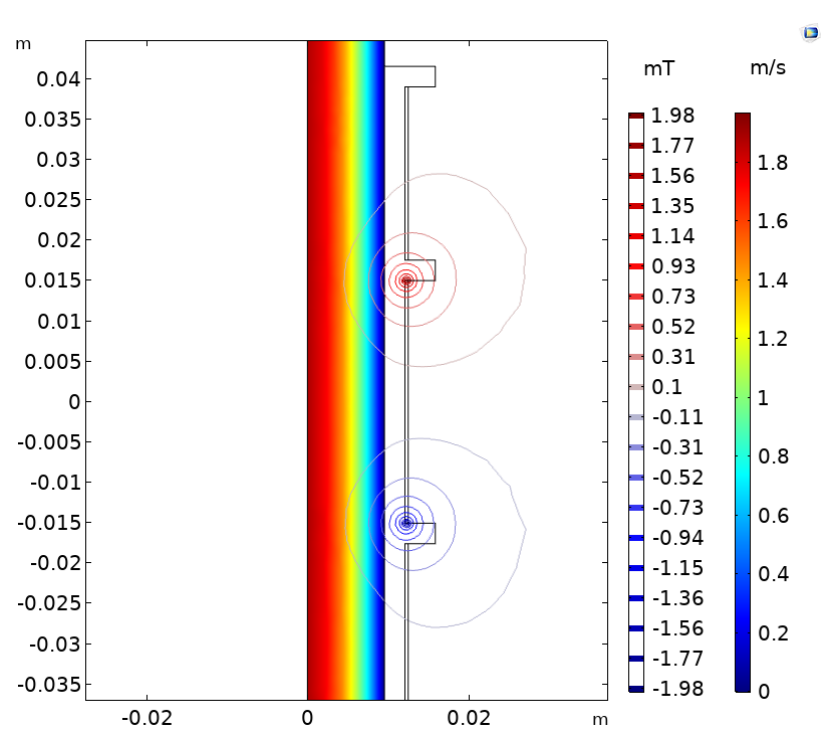

(a) Galinstan

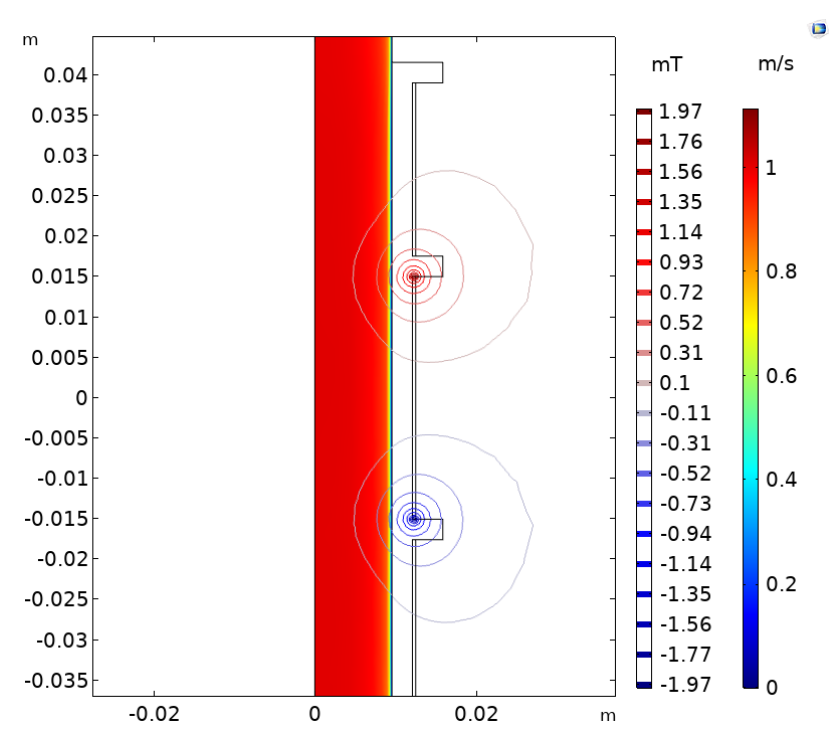

(b) Mercury

Figure 19. Comparison of galinstan and mercury velocity profiles and magnetic flux density $B_{r}(r, z)$.

Figure 20 further verifies the decrease in output voltage from the galinstan simulation. Overall, the ECFM in galinstan is sensing lower velocities given the same inflow velocity as the mercury medium. Hence, lower frequencies must be used to penetrate the magnetic field further into the medium. Calibration can account for the overall measured velocity to a certain point, but considering the discussion regarding skin depth in the aluminum rod simulations, it is known that if the maximum velocity of the fluid is closest to the center of the flow channel, then a lesser output voltage must be measured using the sensor. Also, it cannot be said that the fluid profile given by COMSOL ${ }^{\mathrm{TM}}$ is entirely accurate, as it is a mathematical formulation of ideal fluid flow given the material characteristics of the medium. In comparison, a more accurate picture of the mean velocity flow can be taken with mercury since its predicted flow profile is more uniform. As a reminder, the contour lines are the $B_{r}(r, z)$ component of the magnetic flux density. In Figure 19 , the outer circles are the weakest fields and are tending towards zero, which is gray (between blue and red) on the graphic shown. Red indicates where the magnetic flux density is most positive, and blue shows when it is most negative. As depicted, the maximum magnetic flux densities occur at the inner circles near the gap between the primary and secondary coils. They are opposing in amplitude due to the nature of the additive and opposing magnetic fields caused by the secondary coils as discussed in Section 2.1.

Lastly, the predicted calibration curves for sensor output voltage vs. velocity are shown in Figure 21. Simulations were performed starting at a velocity of $0.1 \frac{\mathrm{m}}{\mathrm{s}}$ opposed to $0 \frac{\mathrm{m}}{\mathrm{s}}$ to help solver convergence. The stationary fluid case will eventually be helpful to compensate for sensor output voltage offsets prior to calibration. 


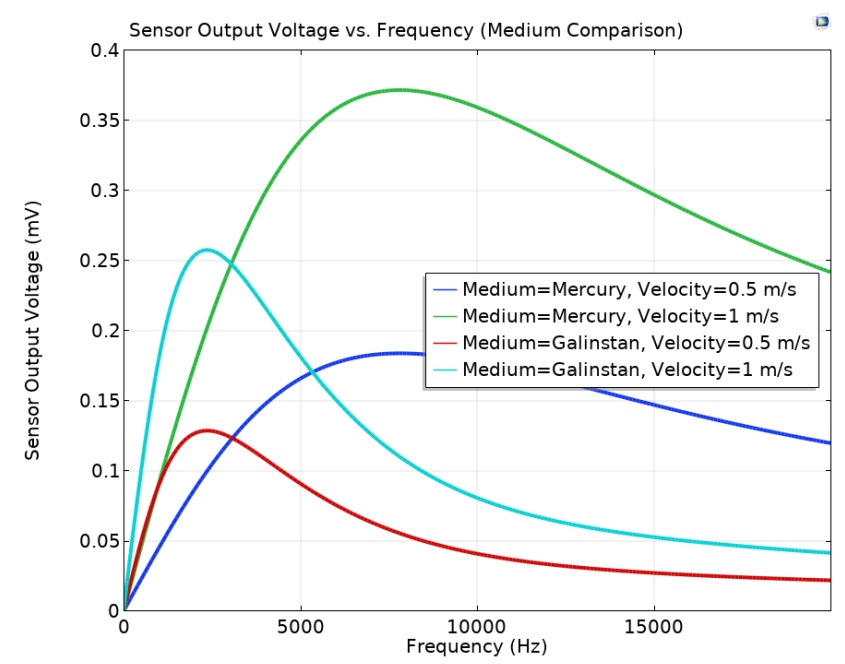

Figure 20. Comparison of frequency response for Mercury and Galinstan.

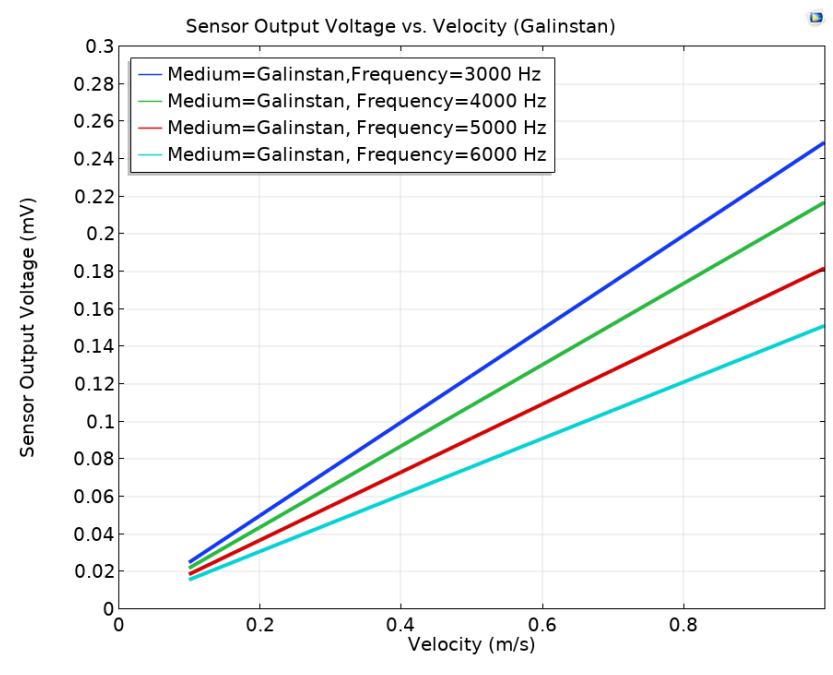

(a) Galinstan

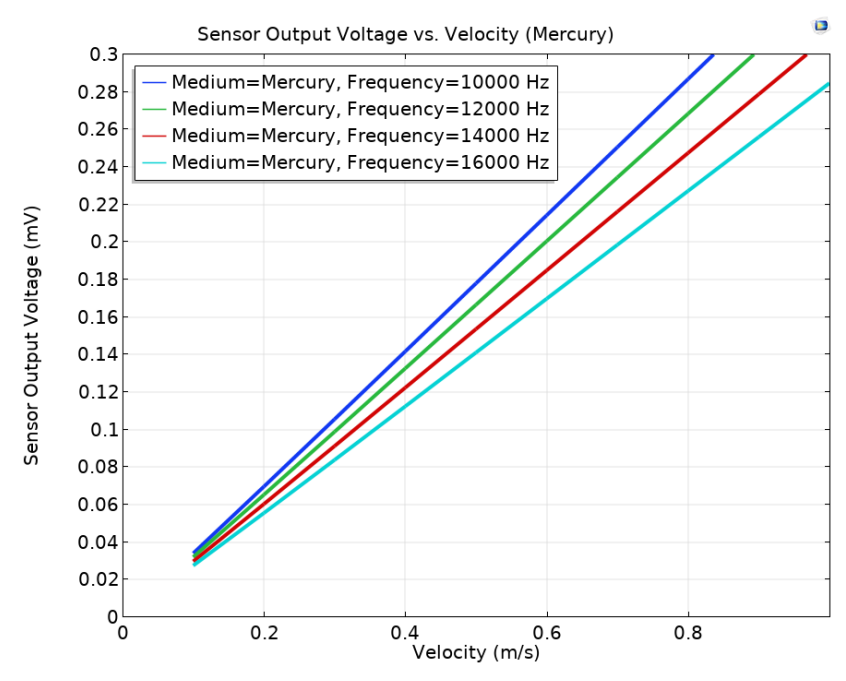

(b) Mercury

Figure 21. Comparison of Gglinstan and Mercury sensor output voltage as a function of velocity. 


\section{CONCLUSIONS}

Characterization of the ECFM has been conducted through simulation, and experiments for the dry testing stage are under way. The effects of the sensor parameters on the sensor output were fully investigated for both dry and liquid test scenarios. It was shown that an optimal excitation frequency can be determined which yields the greatest sensor output for a given geometry. This is crucial in the development of the ECFM, considering space limitations which have yet to be determined for placement in future test assemblies. With the use of the RDT C 4-7T specification and the results presented, methods to actualize the final probe insert sensor were also studied. Furthermore, ECFM simulations in liquid provided insight into the liquid properties for viscosity and conductivity, which significantly effect the sensor's output. By investigating the magnetic flux density and fluid profile overlays, the phenomena which are measured from a liquid coolant flow using the ECFM sensor were revealed.

In summary, it was found that the flow-through type sensor exhibited the following properties:

- Increasing the radius of the flow channel increases sensor output voltage.

- Decreasing the primary and secondary coil length (effectively stacking the coils) increases the sensor output voltage.

- Decreasing the spacing between the primary and secondary coils increases the sensor output voltage.

- Decreasing the conductivity of the flow channel medium by changing the material, increases the excitation signal frequency and produces the largest sensor output voltage for a fixed velocity.

- The sensor output voltage and velocity relationship is linear and can produce calibration curves which predict an unknown velocity for a sensor deployed in the field.

- More viscous fluids reduce the average flow velocity for all excitation signals.

- For a constant fluid velocity, the sensor output voltage always decays after the maximum output frequency is reached.

- The bandwidth of excitation frequencies near the frequency of maximum output voltage for liquid flow channels appears to increase for less viscous and lower conductivity fluids.

Simulation results agree very well with previous experimental trends in terms of the sensor's relation to varying velocity and frequency. This preliminary study has served the purpose of characterizing the sensor's performance based on geometry, optimizing the coil parameters and excitation signals, and generating calibration curves which can now be experimentally tested and verified against alternate flow meters. It is expected that there will be a few iterations of experimental testing and fine tuning of models to achieve a more accurate depiction of the sensor's actual performance. 



\section{REFERENCES}

\section{References}

J. Brewer and R. Jaross. Eddy-current probe type sodium flowsensor for fftf reactor channel flow monitoring. Argonne National Laboratory, 1970.

J. Costello, R. L. Laubham, W. R. Miller, and C. R. Smith. FFTF probetype eddy-current flowmeter: Wet versus dry performance evaluation in sodium. Nuclear Technology, 19(3):174-180, 1973. doi: 10.13182/NT73-3.

P. A. Davidson. An Introduction To Magnetohydrodynamics. Cambridge University Press, The Edinburgh Building, Cambridge CB2 2RU, UK, 2001. ISBN 0521794870.

M. Kumar. Magnetic flux distortion in two-phase liquid metal flow, $\mathrm{PhD}$ Thesis. Dynamique des fluides, Institut National Polytechnique de Toulouse, 2016.

W. T. Nutt and J. L. Stringer. ECFM flow measurements in the FFTF using phase-sensitive detectors. Hanford Engineering Development Laboratory, Provided by PNLL-26202, March 1973.

Standard RDT C 4-7T. Eddy-current probe type flow sensor for liquid metal service. United States Atomic Energy Commission Division of Reactor Research and Development, June 1973.

D. E. Wiegand and C. E. Michels. Performance tests on an eddy-current flowmeter. IEEE Transactions on Nuclear Science, NS-16(2), 1969. 

APPENDIX A. MAXWELL'S EQUATION'S FOR MHD 


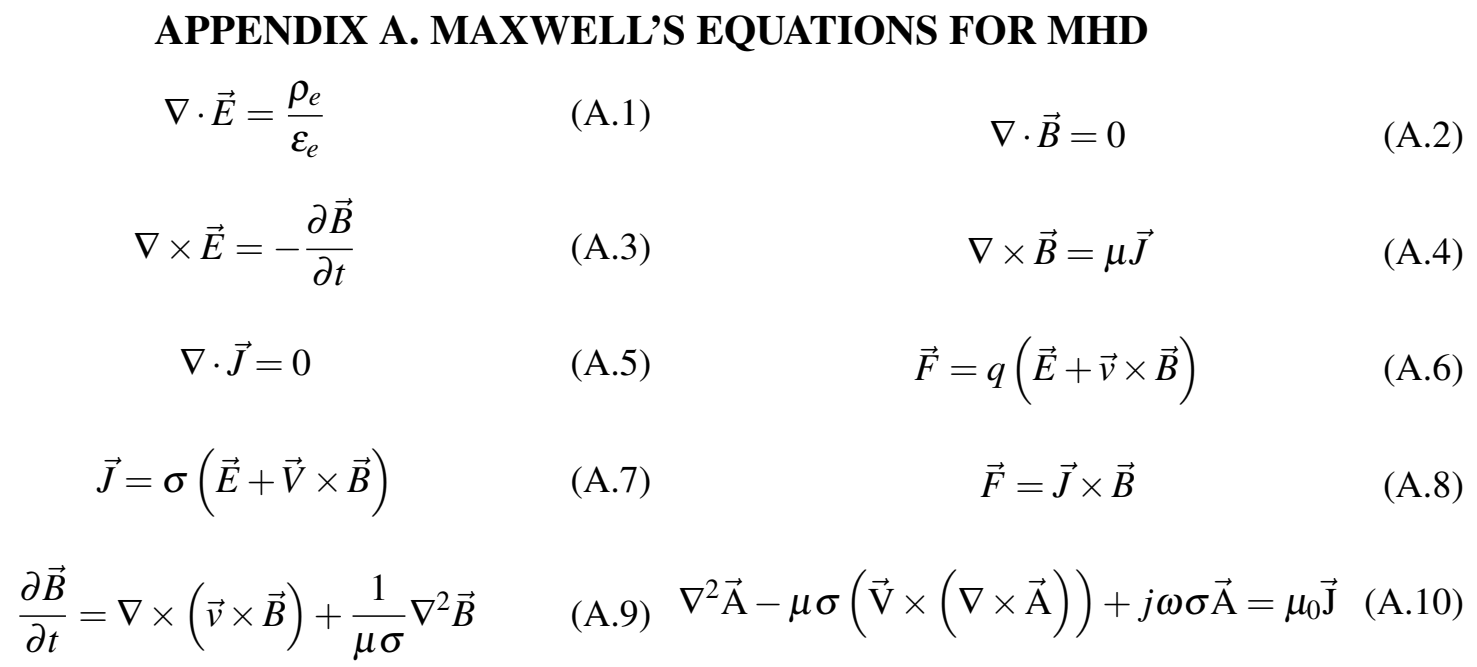

${ }^{*}$ A.10 is the Time Harmonic Advection-Diffusion Equation 